LA-14334

Approved for public release;

distribution is unlimited.

Nonstandard Analysis and Shock Wave Jump Conditions in a One-Dimensional Compressible Gas 
Los Alamos National Laboratory, an affirmative action/ equal opportunity employer, is operated by Los Alamos National Security, LLC, for the National Nuclear Security Administration of the U.S. Department of Energy under contract DE-AC52-06NA25396.

\section{NASS \\ National Nuclear Security Administration}

This report was prepared as an account of work sponsored by an agency of the U.S. Government. Neither Los Alamos National Security, LLC, the U.S. Government nor any agency thereof, nor any of their employees make any warranty, express or implied, or assume any legal liability or responsibility for the accuracy, completeness, or usefulness of any information, apparatus, product, or process disclosed, or represent that its use would not infringe privately owned rights. Reference herein to any specific commercial product, process, or service by trade name, trademark, manufacturer, or otherwise does not necessarily constitute or imply its endorsement, recommendation, or favoring by Los Alamos National Security, LLC, the U.S. Government, or any agency thereof. The views and opinions of authors expressed herein do not necessarily state or reflect those of Los Alamos National Security, LLC, the U.S. Government, or any agency thereof. Los Alamos National Laboratory strongly supports academic freedom and a researcher's right to publish; as an institution, however, the Laboratory does not endorse the viewpoint of a publication or guarantee its technical correctness. 
LA-14334

Issued: May 2007

Nonstandard Analysis and Shock Wave Jump Conditions in a One-Dimensional Compressible Gas

Roy S. Baty

F. Farassat*

John A. Hargreaves

*Aeroacoustics Branch, NASA Langley Research Center, Hampton, VA 23681-2199 



\section{TABLE OF CONTENTS}

1.0 Introduction 1

$\begin{array}{lll}2.0 & \text { Equations of Motion } & 7\end{array}$

$\begin{array}{lll}2.1 & \text { Conservation Equations } & 7\end{array}$

$\begin{array}{lll}2.2 & \text { Inviscid Equations } & 7\end{array}$

2.3 Viscous Equations 9

3.0 Nonstandard Analysis and Hyperreal Numbers 11

3.1 Construction of a Hyperreal Number System 11

3.2 Nonstandard Generalized Functions 19

3.3 Multiplication of Nonstandard Generalized Functions 24

4.0 Inviscid Shock Wave Jump Conditions 30

4.1 Specific Volume, Velocity and Pressure Jump Conditions 30

4.2 Entropy Jump Condition 34

5.0 Viscous Shock Wave Jump Conditions 39

5.1 Jump Conditions for a Viscous Non-Heat Conducting Gas 39

5.2 Jump Conditions for a Viscous Heat Conducting Gas 45

5.3 Comparison of Shock Wave Jump Conditions with Experimental Data 47

6.0 Summary and Conclusions $\quad 49$

$\begin{array}{lll}7.0 & \text { References } & 51\end{array}$

$\begin{array}{lll}8.0 & \text { Figures } & 54\end{array}$ 



\title{
Nonstandard Analysis and Shock Wave Jump Conditions in a One-Dimensional Compressible Gas
}

\author{
Roy S. Baty, F. Farassat, and John A. Hargreaves
}

\begin{abstract}
Nonstandard analysis is a relatively new area of mathematics in which infinitesimal numbers can be defined and manipulated rigorously like real numbers. This report presents a fairly comprehensive tutorial on nonstandard analysis for physicists and engineers with many examples applicable to generalized functions. To demonstrate the power of the subject, the problem of shock wave jump conditions is studied for a one-dimensional compressible gas. It is assumed that the shock thickness occurs on an infinitesimal interval and the jump functions in the thermodynamic and fluid dynamic parameters occur smoothly across this interval. To use conservations laws, smooth pre-distributions of the Dirac delta measure are applied whose supports are contained within the shock thickness. Furthermore, smooth pre-distributions of the Heaviside function are applied which vary from zero to one across the shock wave. It is shown that if the equations of motion are expressed in nonconservative form then the relationships between the jump functions for the flow parameters may be found unambiguously. The analysis yields the classical Rankine-Hugoniot jump conditions for an inviscid shock wave. Moreover, non-monotonic entropy jump conditions are obtained for both inviscid and viscous flows. The report shows that products of generalized functions may be defined consistently using nonstandard analysis; however, physically meaningful products of generalized functions must be determined from the physics of the problem and not the mathematical form of the governing equations.
\end{abstract}

\subsection{INTRODUCTION}

The lion's share of results in modern mathematics have been discovered from the study and application of partial differential equations. Currently, no known mathematical theory provides techniques to study all the linear and nonlinear partial differential equations of mathematical physics. The lack of such a coherent theory makes the analysis of abstract linear partial differential equations very difficult, and the analysis of general nonlinear partial 
differential equations essentially insurmountable. The effect of the absence of a central theory is that each different type of physical problem, and hence, each type of equation, requires a different analytical or numerical method for its solution. Therefore, the subject of partial differential equations reduces to a potpourri of seemingly unrelated techniques.

Existing methods to analyze partial differential equations are not very useful for nonlinear problems, because almost all methods used are based on the linear mathematical structures developed in functional analysis. Since linear mathematical structures do not describe the simplest nonlinearity (multiplication) it is not surprising that functional analysis has not yielded a general framework to study partial differential equations.

Many approaches have been used to attempt to define a useful multiplication operation on spaces of functionals applied in linear analysis to study differential equations. The motivation for multiplying functionals comes from a plethora of applications that occur in modeling physical problems: construction of solutions of certain partial differential equations requires the multiplication of linear functionals. However, a fundamental theorem of Schwartz [1] shows that the standard algebraic product cannot be defined consistently on the spaces of functionals, such as the distributions or classical generalized functions; all such methods will produce ambiguous results leading to logical and arithmetical contradictions. Since standard multiplication does not produce meaningful results, products of generalized functions have been defined which are weaker than standard multiplication. The resulting spaces of generalized functions are algebras containing the classical generalized functions as a proper subset.

Over the last thirty years, E.E. Rosinger [2-5] and J.F. Colombeau [6,7] working independently, have developed a theoretical framework for quotient algebras of generalized functions to analyze nonlinear partial differential equations. Their approach extends classical linear mathematics to nonlinear mathematics by defining multiplication on the classical generalized functions by embedding these linear spaces into abstract algebras of nonlinear generalized functions with a weak operation of multiplication. The potential use of such theories of nonlinear generalized functions allowing the analysis and approximation of solutions to nonlinear partial differential equations is enormous. Such a theory could impact almost all areas of science and technology. In engineering, for example, a coherent mathematical theory would help advance the numerical methods used to predict solutions of complicated problems and could reduce significantly the physical experimentation needed in the solution of engineering problems. 
While it is probable that these theories of generalized functions will yield insight into the theory and computation of certain classes of nonlinear field equations, significant potential limitations of the theory of nonlinear generalized functions are known. Since any logically consistent operation of multiplication of generalized functions must be much weaker than the classical multiplication of numbers and functions, such a product of generalized functions will always yield large collections of abstract objects representing weak solutions of the problem under investigation. The upshot is that a theory of nonlinear generalized functions allows the solution of problems not defined in classical mathematics by relaxing the uniqueness of multiplication. Basically, problems with no classical weak solutions, such as the product $\delta \cdot H$, are converted into problems with uncountably many generalized solutions. The central issue then becomes how to determine a physically observed solution from an infinitude of possible weak solutions.

An example of both the power and limitation of the theory of nonlinear generalized functions can be seen in the research of Rosinger $[5,8]$. In his work, conditions are given which yield generalized solutions for arbitrary polynomial nonlinear partial differential equations. Rosinger has shown that generalized solutions can be obtained for all equations of the form:

$$
\sum_{\alpha} c_{\alpha}(x)\left[\prod_{\beta(\alpha)} \partial^{\beta(\alpha)} U(x)\right]=f(x)
$$

where $U$ is an unknown function $\partial$ is a derivative operator, $\alpha, \beta$ are multi-indices, and $c, f$ are specified functions. However, this result is not immediately useful in practice, because it tells nothing about how to approximate the solution $U$.

To approximate solutions, Colombeau (see references [6,7]) constructed an explicit theory of nonlinear generalized functions. This theory has been applied to analyze and compute solutions to nonlinear partial differential equations. The logic of solving a differential equation in a space of Colombeau generalized functions follows the standard approach of functional analysis: a method is established for generating sequences to approximate solutions within a collection of nonlinear generalized functions and then a compactness argument is applied to show that the approximating sequences actually converge to limits within the space of approximating objects. A resulting limit then represents a solution to the equation. A key difficulty in applying a space of Colombeau generalized functions is determining a specific representation for the products of generalized 
functions occurring in the differential equation. As Colombeau [9] and his co-workers [10,11] have shown, different physical problems require different distinct representations for the same product of generalized functions such as $\delta \cdot H$. The representation of the product is found by adding more physical information about the problem. Roughly speaking, the solution of a concrete problem via nonlinear generalized functions requires: the equations governing the physics, the initial-boundary values, and additional problem-specific physical information required to fix the product of generalized functions. For example, in continuum shock wave problems, the additional information is obtained by requiring the product of generalized functions to satisfy the standard shock wave jump conditions.

In studying the Colombeau generalized functions a number of authors including, Todorov [12], Oberguggenberger [13], and Hoskins and Sousa Pinto [14], have shown that versions of the Colombeau algebras of nonlinear generalized functions may be constructed using nonstandard analysis. Nonstandard analysis is a relatively new area of mathematics which emerged from basic research in mathematical logic. The subject was discovered in the early 1960s by A. Robinson [15]. The main contribution of nonstandard analysis to mathematics is the extension of the real numbers $\mathbf{R}$ to the hyperreal numbers $* \mathbf{R}$ which contain infinitely small (infinitesimal) and infinitely large numbers. The infinitesimals in the hyperreal number system have the properties that Leibniz applied in his development of the calculus, which justifies the algebraic manipulations of infinitesimals that engineers and physicists often use. Moreover, the existence of distinct infinitely large numbers that can be manipulated and used like finite numbers to solve problems provides a powerful new tool for applications. Nonstandard methods may be used to construct algebras of nonlinear generalized functions or nonstandard analysis may be used directly to study problems involving multiplication of generalized functions. The second approach is considered here.

In this report the problem of shock wave jump conditions for a one-dimensional compressible gas is studied using nonstandard analysis. A class of nonstandard Heaviside functions is applied to model the microstructure of the shock wave jump. The term microstructure refers to the analytical description of the functions which represent the flow variables such as density, pressure, velocity and entropy across the shock wave. The shock wave is governed by the standard conservation equations written in primitive form. The shock jump is then modeled to occur over an infinitesimal interval. The family of Heaviside functions applied has some very 
interesting properties which are exploited in the subsequent analysis: for a function $\phi(x)$ with jump $[\phi] \equiv \phi_{r}-\phi_{l}$ at $x=0$ define

$$
\phi(x)=\phi_{l}+[\phi] H(x)
$$

where $\phi(x)$ is at least a $C^{1}$ function and $H(x)$ is a differentiable, nonstandard Heaviside function. Following Colombeau [16], each flow parameter is assumed to have a different Heaviside function associated with it at the shock. This means that each flow variable may have a different shape or microstructure across the shock wave.

It is shown that if the various Heaviside functions for the flow parameters have their jumps located on the same infinitesimal interval, then the governing equations expressed in nonconservative form yield the relations between all these Heaviside functions unambiguously. Strictly speaking, the mathematics of the product of nonstandard generalized functions is unambiguous; that is, use of differentiable nonstandard Heaviside functions removes the mathematical difficulties associated with products such as $\delta \cdot H$. The microstructure of the Heaviside functions predicts the physical microstructure of the shock wave jump if the governing equations of motion model the physics of the shock layer. For an inviscid shock wave, this analysis cannot reproduce the physical microstructure, because the physical information describing the shock region is missing. However, for a viscous shock wave, this analysis reproduces the experimentally observed shock structure. The analysis also yields the classical Rankine-Hugoniot jump conditions found for inviscid compressible flows. Moreover, the unusual microstructure of the Heaviside function for the inviscid entropy jump is obtained by the nonstandard method and is similar to that found by Morduchow and Libby [17] using a viscous flow description and the results of Iollo and Salas [18] using Colombeau's nonlinear generalized functions.

The present work was motivated by the authors' interest (see Baty et al. [19]) in understanding how to add physical information to specify nonlinear generalized functions contained in differential equations which model problems with well defined regions of solution discontinuity, such as a shock wave jump problem. In an intuitive sense Colombeau's generalized functions must represent the infinitesimal microstructure of the shock wave across the jump. Therefore in analogy with these generalized functions, the present work applies nonstandard analysis to build a rigorous, explicit example of the infinitesimal microstructure of a shock wave. 
If the existence of the nonstandard Heaviside functions is assumed given, the present development greatly simplifies the description of the product of generalized functions, because the level of mathematical abstraction is reduced to an advanced calculus problem and not that of a research problem in functional analysis, Keisler [20] and Laugwitz [21]. Moreover, the derivation of the hyperreal shock wave jump conditions helps distinguish between mathematical and physical ambiguities in the inviscid and viscous flow cases.

Chapter 2 begins the technical development of the report by summarizing the equations of motion in nonconservative form for a compressible perfect gas in one spatial dimension. Equations for the inviscid and viscous flow cases as well as the non-heat conducting and heat conducting cases are given. The basic physical assumptions about the gas are also outlined.

Chapter 3 outlines a generic construction of the hyperreal number system. Examples of nonstandard differentiable Heaviside functions are then introduced. Some basic properties of the nonstandard Heaviside functions are reviewed and examples of products of these nonstandard jump functions and their derivatives are exhibited in the context of products of generalized functions.

Chapter 4 applies nonstandard differentiable Heaviside functions to derive shock wave jump conditions for an inviscid compressible gas. The relations between the jump functions are obtained and then used to show that the entropy must exhibit a peak in the shock layer. The location of the entropy peak is found to depend on the Heaviside functions for the specific volume and pressure and cannot be specified for an inviscid flow field.

Chapter 5 extends the derivation of shock wave jump conditions of Chapter 4 to a viscous compressible gas and shows that the flow field Heaviside functions are specified by the viscous flow assumption. The shock wave jump functions are computed for both the non-heat conducting and heat conducting cases for low Mach numbers. The computed Heaviside function for density is also compared with the density measured for a normal shock wave in a low density helium flow field.

Chapter 6 then concludes the report with a summary of the key observations and results presented. 


\subsection{EQUATIONS OF MOTION}

\subsection{Conservation Equations}

The Navier-Stokes equations govern the motion of compressible fluid flow. In Cartesian coordinates $\left(x^{1}, x^{2}, x^{3}\right)$, the equations governing conservation of mass, momentum and energy are given by:

$$
\begin{gathered}
\frac{\partial \rho}{\partial t}+u^{k} \frac{\partial \rho}{\partial x^{k}}+\rho \frac{\partial u^{k}}{\partial x^{k}}=0 \\
\rho \frac{\partial u^{j}}{\partial t}+\rho u^{k} \frac{\partial u^{j}}{\partial x^{k}}=-\frac{\partial p}{\partial x^{j}}+\frac{\partial}{\partial x^{j}}\left(\lambda \frac{\partial u^{k}}{\partial x^{k}}\right)+\frac{\partial}{\partial x^{i}}\left[\mu\left(\frac{\partial u^{i}}{\partial x^{j}}+\frac{\partial u^{j}}{\partial x^{i}}\right)\right] \\
\rho \frac{\partial e}{\partial t}+\rho u^{k} \frac{\partial e}{\partial x^{k}}=-p \frac{\partial u^{k}}{\partial x^{k}}+\frac{\partial}{\partial x^{j}}\left(k \frac{\partial T}{\partial x^{j}}\right)+\lambda\left(\frac{\partial u^{k}}{\partial x^{k}}\right)^{2}+\mu\left(\frac{\partial u^{i}}{\partial x^{j}}+\frac{\partial u^{j}}{\partial x^{i}}\right) \frac{\partial u^{j}}{\partial x^{i}},
\end{gathered}
$$

where $u^{k}(k=1,2,3)$ are the velocity components, $\rho$ is the density, $p$ is the pressure, $e$ is the energy per unit mass, and $T$ is the temperature. The parameters $\mu, \lambda$ and $k$ are the dynamic viscosity, the second viscosity coefficient and, the thermal conductivity of the fluid, respectively. These parameters are assumed to be constant. The conservation equations (2.1), (2.2) and (2.3) represent five equations in terms of seven physical variables. To close the system of equations two equations of state must be added which relate the thermodynamic variables $\rho, p, e$ and $T$ :

$$
\begin{aligned}
& p=p(\rho, T), \\
& e=e(\rho, T) .
\end{aligned}
$$

Developments of equations (2.1), (2.2), and (2.3) and the equations of state (2.4) and (2.5) may be found in Currie [22] and Thompson [23].

\subsection{Inviscid Equations}

For the development of the shock wave jump conditions for an inviscid, non-heat conducting gas the conservation equations are assumed to model the motion of a gas in one spatial dimension and time:

$$
\left(u^{1}, u^{2}, u^{3}\right)=(u, 0,0) \text { so that } u \equiv u^{1} \text { and } x \equiv x^{1} .
$$


Furthermore, since the gas is assumed to be inviscid and non-heat conducting the fluid parameters drop out:

$$
\mu=0, \lambda=0 \text {, and } k=0 .
$$

With these assumptions, equations (2.1) to (2.3) become

$$
\begin{gathered}
\frac{\partial \rho}{\partial t}+u \frac{\partial \rho}{\partial x}+\rho \frac{\partial u}{\partial x}=0, \\
\rho \frac{\partial u}{\partial t}+\rho u \frac{\partial u}{\partial x}=-\frac{\partial p}{\partial x}, \\
\rho \frac{\partial e}{\partial t}+\rho u \frac{\partial e}{\partial x}=-p \frac{\partial u}{\partial x} .
\end{gathered}
$$

For a perfect gas, the equations of state are given by

$$
\begin{gathered}
e=c_{v} T, \\
p=\rho R T,
\end{gathered}
$$

where $c_{v}$ is the specific heat at constant volume (assumed constant) and $R$ is the specific gas constant.

The conservation equations are expressed in terms of specific volume $v \equiv \frac{1}{\rho}$ rather than the density. Also, the notation for partial differentiation is simplified to the subscript notation, $\xi_{x} \equiv \frac{\partial \xi}{\partial x}$. With these assumptions equations (2.7) - (2.9) become:

$$
\begin{gathered}
v_{t}-v u_{x}+u v_{x}=0, \\
u_{t}+u u_{x}+v p_{x}=0, \\
e_{t}+u e_{x}+v p u_{x}=0 .
\end{gathered}
$$

Next combining the equations of state with the energy equation (2.14), it may be shown that

$$
p\left(s_{t}+u s_{x}\right)=c_{v}\left(p_{t}+u p_{x}+\gamma p u_{x}\right)
$$

where

$$
s=c_{v} \log \left(p v^{\gamma}\right),
$$


is the entropy per unit mass and where $\gamma$ is the ratio of specific heats. Since the gas motion on either side of a shock wave is assumed to be isentropic, equation (2.15) reduces to zero along streamlines upstream and downstream of a shock wave. However, a shock wave is not an isentropic process, so across the shock wave equation (2.15) is not zero:

$$
p\left(s_{t}+u s_{x}\right) \neq 0
$$

which implies

$$
c_{v}\left(p_{t}+u p_{x}+\gamma p u_{x}\right) \neq 0
$$

Therefore, the equations governing the unsteady, one-dimensional motion of an inviscid, non-heat conducting gas across a shock wave are given by:

$$
\begin{gathered}
v_{t}-v u_{x}+u v_{x}=0, \\
u_{t}+u u_{x}+v p_{x}=0, \\
s=c_{v} \log \left(p v^{\gamma}\right) .
\end{gathered}
$$

\subsection{Viscous Equations}

For the development of the shock wave jump conditions for an viscous, heat conducting gas the conservation equations are assumed again to model the motion of a gas in one spatial dimension and time. Applying the conditions of expression (2.6) to equations (2.1) through (2.3) then yields

$$
\begin{gathered}
\frac{\partial \rho}{\partial t}+u \frac{\partial \rho}{\partial x}+\rho \frac{\partial u}{\partial x}=0 \\
\rho \frac{\partial u}{\partial t}+\rho u \frac{\partial u}{\partial x}=-\frac{\partial p}{\partial x}+\frac{\partial}{\partial x}\left(\lambda \frac{\partial u}{\partial x}\right)+\frac{\partial}{\partial x}\left[2 \mu \frac{\partial u}{\partial x}\right], \\
\rho \frac{\partial e}{\partial t}+\rho u \frac{\partial e}{\partial x}=-p \frac{\partial u}{\partial x}+\frac{\partial}{\partial x}\left(k \frac{\partial T}{\partial x}\right)+\lambda\left(\frac{\partial u}{\partial x}\right)^{2}+2 \mu\left(\frac{\partial u}{\partial x}\right)^{2} .
\end{gathered}
$$

And assuming the Stokes' relation holds

$$
\lambda=-\frac{2}{3} \mu
$$

equations (2.21) and (2.22) become 


$$
\begin{gathered}
\rho \frac{\partial u}{\partial t}+\rho u \frac{\partial u}{\partial x}=-\frac{\partial p}{\partial x}+\frac{4}{3} \frac{\partial}{\partial x}\left[\mu \frac{\partial u}{\partial x}\right], \\
\rho \frac{\partial e}{\partial t}+\rho u \frac{\partial e}{\partial x}=-p \frac{\partial u}{\partial x}+\frac{\partial}{\partial x}\left(k \frac{\partial T}{\partial x}\right)+\frac{4}{3} \mu\left(\frac{\partial u}{\partial x}\right)^{2} .
\end{gathered}
$$

Next, expressing the conservation equations (2.20), (2.24) and (2.25) in terms of the specific volume $v=\frac{1}{\rho}$ rather than the density, and applying the subscript notation for partial differentiation yields:

$$
\begin{gathered}
v_{t}-v u_{x}+u v_{x}=0, \\
u_{t}+u u_{x}+v p_{x}-\frac{4}{3} v\left(\mu u_{x}\right)_{x}=0, \\
e_{t}+u e_{x}+v p u_{x}-v\left(k T_{x}\right)_{x}-\frac{4}{3} \mu v\left(u_{x}\right)^{2}=0 .
\end{gathered}
$$

Now, from the equations of state (2.10) and (2.11)

$$
e=p v \cdot \frac{c_{v}}{R}
$$

Recall from thermodynamics that

$$
R=c_{p}-c_{v},
$$

which yields

$$
e=\frac{p v}{\gamma-1}
$$

where

$$
\gamma=\frac{c_{p}}{c_{v}}
$$

Combining equations (2.28) and (2.29) then produces the following form of the energy equation:

$$
\frac{1}{\gamma-1} \cdot\left[(p v)_{t}+u(p v)_{x}\right]+v p u_{x}-v\left(k T_{x}\right)_{x}-\frac{4}{3} \mu v\left(u_{x}\right)^{2}=0
$$

Equations (2.26), (2.27) and (2.30) are used to study shock jump conditions in an unsteady, onedimensional, viscous, heat conducting, perfect gas. 


\subsection{NONSTANDARD ANALYSIS AND HYPERREAL NUMBERS}

\subsection{Construction of a Hyperreal Number System}

A construction of a nonstandard or hyperreal number system * $\mathbf{R}$ is outlined in this section. The construction is roughly analogous to the construction of the real numbers $\mathbf{R}$ using equivalence classes of Cauchy sequences of rational numbers. For the hyperreal numbers, however, the equivalence relation is based on the rates of convergence of sequences of real numbers as well as the limits of convergence. The present notation and development essentially repeat that of Hurd and Loeb [24]. Excellent elementary introductions to nonstandard analysis may be found in Henle and Kleinberg [25], Keisler [20,26] and Hoskins [27], while more advanced developments may be found in Lindstrom [28], Stroyan and Luxemburg [29], Davis [30], and Arkeryd, et al. [31].

The goal of a construction of a hyperreal number system is to produce a linearly ordered field $(* \mathbf{R},+, ;<)$ which contains an isomorphic copy of the linearly ordered field of real numbers, but also contains infinitely large and infinitely small elements. Let $N$ be the set of natural numbers $1,2,3, \ldots$. And let $\boldsymbol{R}_{\boldsymbol{s}}$ be the set of all sequences of real numbers indexed by $N$. Every element $r \in \boldsymbol{R}_{\boldsymbol{s}}$ many be written as:

$$
r=\left\{r_{1}, r_{2}, r_{3}, \ldots\right\}=\left\{\left(r_{n}\right)\right\}
$$

Natural componentwise operations of addition and multiplication may be defined for all $r, s \in \boldsymbol{R}_{\boldsymbol{s}}$ via

$$
r \oplus s=\left\{\left(r_{n}\right)\right\} \oplus\left\{\left(s_{n}\right)\right\}=\left\{\left(r_{n}+s_{n}\right)\right\}
$$

and

$$
r \otimes s=\left\{\left(r_{n}\right)\right\} \otimes\left\{\left(s_{n}\right)\right\}=\left\{\left(r_{n} \cdot s_{n}\right)\right\}=\left\{\left(r_{n} s_{n}\right)\right\} .
$$

With these binary operations, the resulting 3-tuple is a commutative ring with the identity:

$$
1=\{1,1,1,1, \ldots\}
$$

and the zero

$$
0=\{0,0,0,0, \ldots\}
$$


As an example of the algebraic operations defined by equations (3.2) and (3.3), consider the sequences

$$
r=\{1,3,5,7, \ldots\} \text { and } s=\{2,4,6,8, \ldots\} .
$$

Then $r \oplus s$ and $r \otimes s$ yield:

$$
r \oplus s=\{3,7,11,15, \ldots\}
$$

and

$$
r \otimes s=\{2,12,30,56, \ldots\} .
$$

All algebraic concepts used in the subsequent development may be found in Hungerford [32].

The space of all real sequences with the above addition and multiplication does not form a field, because of the existence of non-zero zero divisors, for example:

$$
\{1,0,1,0,1, \ldots\} \otimes\{0,1,0,1,0, \ldots\}=\{0,0,0,0, \ldots\}=0 .
$$

This problem is repaired by introducing an equivalence relation $\sim$ on $\boldsymbol{R}_{\boldsymbol{s}}$ and defining algebraic and order structures on a quotient space $\boldsymbol{R}_{\boldsymbol{s}} / \sim$ to yield a linearly ordered field $(* \mathbf{R},+, \cdot,<)$. The equivalence relation is defined using the concept of an ultrafilter.

Definition 1. Let $I$ be a nonempty set. A filter on $I$ is a nonempty collection $U$ of subsets of $I$ having the following properties:

a. The empty set is not contained in $U: \varnothing \notin U$,

b. If $A, B \in U$, then $A \cap B \in U$,

c. If $A \in U$ and $I \supseteq B \supseteq A$ then $B \in U$.

Definition 2. A filter is called an ultrafilter if for any subset $A$ of $I$ either $A \in U$ or its complement $A^{c}=I-A \in U$.

Definition 3. If $I$ is an infinite set, the collection of subsets $F_{I}=\{A \subseteq I \mid I-A$ is finite $\}$ is a filter called the cofinite filter on $I$. More generally, set $A$ is cofinite with respect to $I$ if $I-A$ is finite. An ultrafilter $U$ on $I$ is free if it contains $F_{I}$. 
A free ultrafilter $U$ cannot contain any finite set, or else the free ultrafilter $U$ must contain the empty set and violates Definition 1a. The existence of free ultrafilters is not obvious and not trivial. Their existence follows from a form of the axiom of choice and is highly nonconstructive. Henle and Kleinberg [25] show how to obtain the elements of an ultrafilter on $N$ using transfinite induction. The following theorem guaranteeing the existence of free ultrafilters is proved in Hurd and Loeb [24, p221]:

Theorem 4. Free ultrafilters exist on any infinite set $I$.

Now that it is known that free ultrafilters may be shown to exist on any infinite set, select any free ultrafilter $U$ on the set of natural numbers $N$. $U$ will contain all the cofinite sets of $N$. A relation $\sim{ }_{U}$ is defined on the set of all real sequences of numbers, $\boldsymbol{R}_{\boldsymbol{s}}$ using the ultrafilter $U$. To simplify the notation the relation is written as ' $\sim$.'

Definition 5. If $r, s \in \boldsymbol{R}_{s}$, then $r \sim s$ if and only if $\left\{n \in N \mid r_{n}=s_{n}\right\} \in U$.

Lemma 6. The relation $\sim$ is an equivalence relation on $\boldsymbol{R}_{\boldsymbol{s}}$.

The proof of Lemma 6 is given in Hurd and Loeb [24, p4]. Since the relation $\sim$ forms an equivalence relation on $\boldsymbol{R}_{\boldsymbol{s}}, \boldsymbol{R}_{\boldsymbol{s}}$ is cut up into disjoint subsets or equivalence classes. Each equivalence class contains all the sequences of real numbers that agree on infinite indexing subsets of $N$ which are contained in the free ultrafilter $U$. Following Definition 5 , if

$$
r=\{100,-5,7,16,16,16, \ldots\} \text { and } s=\{-18,11,14,15,16,16,16, \ldots\},
$$

we have $r \sim s$, because the set $\left\{n \in N \mid r_{n}=s_{n}\right\}=\{5,6,7,8, \ldots\} \in U$ is cofinite with respect to $N$. The equivalence relation $\sim$ is now used to define the nonstandard or hyperreal numbers.

Definition 7. Let * $\mathbf{R}$ denote the set of all equivalence classes of $\boldsymbol{R}_{\boldsymbol{S}}$ induced by $\sim$ :

$$
* \mathbf{R} \equiv \boldsymbol{R}_{s} / \sim .
$$


The equivalence class containing a given sequence $s \in \boldsymbol{R}_{\boldsymbol{s}}$ is denoted by [s]. Therefore, if $r \sim s$ in $\boldsymbol{R}_{\boldsymbol{s}}$ then

$$
[r]=[s] \text { in } * \mathbf{R} \text {. }
$$

The elements of $* \mathbf{R}$ are called the nonstandard or hyperreal numbers.

From the construction of $* \mathbf{R}$ via the free ultrafilter $U$ it may be shown that either $\{1,0,1,0,1, \ldots\}$ or $\{0,1,0,1,0, \ldots\}$ but not both, will be identified with the zero element $[0]$ in $* \mathbf{R}$. Therefore, the ultrafilter removes the problem of non-zero zero divisors in the commutative ring based on the set of all real sequences, $\boldsymbol{R}_{\boldsymbol{s}}$. Since the selection of the free ultrafilter $U$ determines which sequence $\{1,0,1,0,1, \ldots\}$ or $\{0,1,0,1,0, \ldots\}$ is identified with zero, the construction of the nonstandard numbers depends on $U$ and does not appear to be unique. Uniqueness of the hyperreals turns out to be a deep and subtle issue. It has been proved that if the continuum hypothesis holds, the construction of the hyperreal numbers $* \mathbf{R}$ is independent of the selection of the free ultrafilter in the following sense: all such quotient fields constructed from a free ultrafilter based on $N$ are isomorphic ordered fields. This means that one realization of $* \mathbf{R}$ is algebraically equivalent to any other realization of $* \mathbf{R}$. However, the continuum hypothesis is not decidable within the Zermelo-Fraenkel axioms of set theory. If the continuum hypothesis is taken as an axiom, the hyperreals are unique; on the other hand without the continuum hypothesis, the uniqueness of the hyperreals is not decidable, Goldblatt [33].

Next to produce a linearly ordered field $(* \mathbf{R},+, ;,<)$, the algebraic and order relations must be defined.

Definition 8. Let $[r],[s] \in * \mathbf{R}$. Then define (using equations $3.1-3.3$ ):
a. $[r]+[s]=[r \oplus s]=\left[\left\{\left(r_{n}+s_{n}\right)\right\}\right]$,
b. $[r] \cdot[s]=[r][s]=[r \otimes s]=\left[\left\{\left(r_{n} s_{n}\right)\right\}\right]$,
c. $[r]<[s]$ if and only if $\left\{n \in N \mid r_{n}<s_{n}\right\} \in U$, and
d. $[r] \leq[s]$ if and only if $[r]<[s]$ or $[r]=[s]$, where $>$ and $\geq$ are defined in a similar way. 
The construction of * $\mathbf{R}$ together with the operations of Definition 8 yield a linearly ordered field:

Theorem 9. $\left({ }^{*} \mathbf{R},+, ;,<\right)$ is a linearly ordered field.

The proof of Theorem 9 is simple; it is outlined in Hurd and Loeb [24, pp5-6].

Notice that the real numbers $\mathbf{R}$ form a linearly ordered subfield of $\left({ }^{*} \mathbf{R},+, ;<\right)$. This may be seen by introducing a mapping

$$
*: \mathbf{R} \rightarrow * \mathbf{R} \text {, }
$$

to embed $\mathbf{R}$ into * $\mathbf{R}$.

Definition 10. If $r \in \mathbf{R}$ define the mapping of expression 3.5 by

$$
*(r)=* r \equiv[\{r, r, r, \ldots\}] \in * \mathbf{R} .
$$

Theorem 11. The * mapping of Definition 10 is an order preserving isomorphism of $\mathbf{R}$ into $* \mathbf{R}$.

The proof of Theorem 11 is a straightforward verification of the relevant definitions.

Definition 12. If $A \subseteq \mathbf{R}$, then $A^{*}$ is the set of all elements *a, where $a \in A . \mathbf{R}^{*}$ is the set of standard numbers in * $\mathbf{R}$.

Definition 13. Denote a hyperreal number by $s$ (rather than $[s]$ ).
a. A number $s \in * \mathbf{R}$ is infinite if $n<|s|$ for all standard natural numbers $n$;
b. A number $s \in * \mathbf{R}$ is finite if $|s|<n$ for some standard natural number $n$;
c. A number $s \in * \mathbf{R}$ is infinitesimal if $|s|<\frac{1}{n}$ for all standard natural numbers $n$.

At this stage, true infinitesimals may be identified in $* \mathbf{R}$ which do not exist in $\mathbf{R}$. For example, 


$$
\varepsilon=\{1,1 / 2,1 / 3,1 / 4, \ldots\}=\left\{\left(\frac{1}{n}\right)\right\}
$$

is an infinitesimal since given any non-zero and finite hyperreal number $r=\{(r)\}$, which is also a real number, $\varepsilon<r$ no matter how small $r$ is. This is true, because if $n_{0}=[1 / r]$, the smallest natural number which is greater than or equal to $1 / r$, then

$$
\left\{n \in N \mid \frac{1}{n}<r\right\}=\left\{n_{0}, n_{0}+1, n_{0}+2, \ldots\right\} \in U .
$$

Note that the infinite sequence defining $\varepsilon$ is $\{(1 / n)\}$. Therefore, $* \mathbf{R}$ is much larger than $\mathbf{R}$, containing infinitely small numbers which can be manipulated like finite numbers. Moreover, consider the hyperreal number

$$
\Omega=\{1,2,3,4, \ldots\}=\{(n)\} .
$$

This number is larger than any finite number $p=\{(p, p, p, \ldots)\}$ and is therefore an infinite hyperreal number because the set $\{n \in N \mid n>p\} \in U$. Note that

$$
\begin{gathered}
\Omega+1=\{2,3,4,5,6, \ldots\}=\{(n+1)\}, \\
\frac{\Omega}{2}=\left\{\frac{1}{2}, 1, \frac{3}{2}, 2, \frac{5}{2}, \ldots\right\}=\left\{\left(\frac{n}{2}\right)\right\}, \text { and } \\
\varepsilon \Omega=\left\{1 \cdot 1, \frac{1}{2} \cdot 2, \frac{1}{3} \cdot 3, \frac{1}{4} \cdot 4, \ldots\right\}=\left\{\left(\frac{1}{n} \cdot n\right)\right\}=1 .
\end{gathered}
$$

Also note that $\Omega^{2}=\left\{1^{2}, 2^{2}, 3^{2}, 4^{2}, \ldots\right\}=\left\{\left(n^{2}\right)\right\}>\Omega$ gives another distinct infinite number. Notice that each finite hyperreal number is either equal to a real number or else it is infinitesimally close to a real number.

Definition 14. A finite real number which is infinitesimally close to a finite hyperreal number is called the standard part of $[a]$, written st $(a)$ or just $a$.

For example if $a=\{(1+1 / n)\}$, then st $(a)=\{(1,1,1, \ldots)\}=1$. 
Definition 15. Let $x, y \in * \mathbf{R}$.
a. $x$ and $y$ are infinitesimally close if $x-y$ is an infinitesimal, written $x \approx y$. The monad of $x$ is the set $m(x)=\{y \in * \mathbf{R} \mid x \approx y\}$.
b. $x$ and $y$ are finitely close if $x-y$ is finite.

As implied by the examples above, a hyperreal number system contains many sets which are not subsets of the real numbers. The subsets of a hyperreal number system * $\mathbf{R}$ may be classified into two types of subsets: the internal and the external subsets. The internal subsets are the elements of standard subsets of $\mathbf{R}$ embedded into * $\mathbf{R}$ by the * mapping of Definition 10 .

Definition 16. A subset $A^{*} \subset * \mathbf{R}$ is called internal if there exists a sequence $\left\{\left(A_{n}\right)\right\}$ of subsets of $\mathbf{R}$ such that

$$
x=\left\{\left(x_{n}\right)\right\} \in A^{*} \text { if and only if }\left\{n \in N \mid x_{n} \in A_{n}\right\} \in U
$$

A set $\mathfrak{I} \subset * \mathbf{R}$ is external if it is not internal.

The external objects contained in $* \mathbf{R}$ are the objects which do not result from objects in standard analysis, while the internal objects are precisely the objects which result from extending standard analysis via the * mapping.

Definition 17. Let $\left\{\left(f_{n}\right)\right\}$ be a sequence of functions defined on a set $A \subset \mathbf{R}$ :

$$
f_{n}: A \rightarrow \mathbf{R}
$$

Then an internal function $F$

$$
F: A^{*} \rightarrow * \mathbf{R}
$$

is defined by the assignment

$$
x=\left\{\left(x_{n}\right)\right\} \in A^{*} \rightarrow F(x) \in * \mathbf{R},
$$

where $A^{*} \subset * \mathbf{R}$ and

$$
F(x)=\left\{\left(f_{n}\right)\right\} \bullet\left\{\left(x_{n}\right)\right\}=\left\{\left(f_{n}\left(x_{n}\right)\right)\right\} .
$$


Example 18. As an example of an internal function, consider the sequence of functions

$$
\left\{f_{n}(x)\right\}=\left\{\frac{1}{\pi} \cdot \frac{n}{1+n^{2} x^{2}}\right\} .
$$

An internal function $F(x)$ results for each hyperreal number $\left\{\left(x_{n}\right)\right\}$ :

$$
F(x)=\left\{\left(\frac{1}{\pi} \cdot \frac{n}{1+n^{2} x_{n}^{2}}\right)\right\} .
$$

If the sequences of real functions used to obtain an internal function $F(x)$ are componentwise differentiable and integrable, the internal function will be differentiable and integrable via

$$
\frac{d F}{d x} \equiv\left\{\left(f_{n}^{\prime}\right)\right\}
$$

and

$$
\int_{a}^{x} F(\tau) d \tau \equiv\left\{\left(\int_{a_{n}}^{x_{n}} f_{n}(\tau) d \tau\right)\right\}
$$

where $x=\left\{\left(x_{n}\right)\right\}$ and $a=\left\{\left(a_{n}\right)\right\}$.

Moreover, calculus over the hyperreal numbers is straightforward and is analogous to calculus over the real numbers. As examples, the derivative at $x=a, a \in \mathbf{R}$, of a differentiable function $f(x)$ is defined as

$$
\left.\frac{d f}{d x}\right|_{a}=\operatorname{st}\left(\frac{* f(x)-* f(a)}{x-a}\right),
$$

where $x \approx a$ and $x \neq a$; and the Reimann integral is given by the hyperreal sum

$$
\int_{a}^{b} f(x) d x=\operatorname{st}\left(\sum_{k=1}^{\Omega} * f\left(\xi_{k}\right) \Delta x_{k}\right)
$$

where $\Omega=[(n)]$. See Keisler [20,26] and Henle and Kleinberg [25] for a development of the basic calculus in terms of the hyperreal numbers. 
The * mapping has an important property that is key to applications of nonstandard analysis to standard real analysis. Suppose a logical sentence, denoted by $\Phi$, is defined to be a formula in a first-order language for the real numbers. The truth of a sentence $\Phi$ is conserved under the * mapping in a strong sense: $* \Phi$ is true if and only if $\Phi$ is true. In practice this result means that theorems in real analysis are proved if nonstandard versions of the theorems are proved; that is, standard results follow from nonstandard proofs. This conservation property of the * mapping is called the transfer principle and follows from a theorem in mathematical logic on ultrafilter extensions. A proof of the transfer principle is developed in Hurd and Loeb [24].

\subsection{Nonstandard Generalized Functions}

In the early development of nonstandard analysis, hyperreal techniques were applied to the classical generalized functions, Robinson [15], with application to quantum theory, Kelemen and Robinson [34]. Robinson noticed that any regularizing sequence of smooth functions used to represent the Dirac delta measure could be replaced by a single function with hyperreal parameters. Intuitively a single function represents the limit of the regularizing sequence. Consider the following delta sequence,

$$
s_{n}(x)=\frac{1}{\pi} \cdot \frac{n}{1+n^{2} x^{2}} .
$$

Then letting the limiting parameter $n$ be any infinite hyperreal number $n \rightarrow \Omega$, a smooth nonstandard function acting like the Dirac delta results:

$$
\delta(x)=\frac{1}{\pi} \cdot \frac{\Omega}{1+\Omega^{2} x^{2}} .
$$

This nonstandard delta measure behaves exactly like its classical counterpart: taking the standard part of the integral yields a functional producing the pointwise evaluation,

$$
\lim _{n \rightarrow \infty} \int_{-\infty}^{\infty} s_{n}(x) \phi(x) d x=\mathrm{st}\left(\int_{-\infty}^{\infty} \frac{1}{\pi} \cdot \frac{\Omega}{1+\Omega^{2} x^{2}} \cdot \phi(x) d x\right)=\phi(0)
$$

for an arbitrary test function $\phi \in D(\mathbf{R})$. More generally, the right-hand-side of equation (3.10) may be used to define nonstandard linear functionals on spaces of internal test functions $\phi \in D(* \mathbf{R})$. 
A nonstandard Dirac delta measure may be defined for any standard function $g$ which satisfies

$$
\int_{-\infty}^{\infty} g(x) d x=1
$$

by considering the internal function

$$
\delta(x)=\Omega \cdot g(\Omega \cdot x)
$$

where $\Omega$ is any infinite hyperreal number, Laugwitz [35].

Example 19. To see how the internal function of equation (3.9) acts like the Dirac delta, perform the integration:

$$
\int_{-\infty}^{\infty} \frac{1}{\pi} \cdot \frac{\Omega}{1+\Omega^{2} x^{2}} \cdot \phi(x) d x \approx \frac{\phi(0)}{\pi} \cdot \int_{-\varepsilon(\Omega)}^{\varepsilon(\Omega)} \frac{\Omega}{1+\Omega^{2} x^{2}} d x=\left.\frac{\phi(0)}{\pi} \cdot \arctan (\Omega x)\right|_{-\varepsilon(\Omega)} ^{\varepsilon(\Omega)},
$$

where $\varepsilon=\varepsilon(\Omega)$ is any infinitesimal such that $\Omega \varepsilon$ is an infinite hyperreal satisfying

$$
\arctan (\Omega \varepsilon) \approx \frac{\pi}{2}
$$

Hence,

$$
\int_{-\infty}^{\infty} \frac{1}{\pi} \cdot \frac{\Omega}{1+\Omega^{2} x^{2}} \cdot \phi(x) d x \approx \frac{\phi(0)}{\pi} \cdot\left(\frac{\pi}{2}-\left(-\frac{\pi}{2}\right)\right)=\phi(0) .
$$

Then taking the standard part of the integral yields

$$
\operatorname{st}(\phi(0))=\phi(0),
$$

which shows that the internal function of (3.9) acts like the delta measure.

The power of the linear theory of generalized functions comes from the fact that many distinct regularizing sequences of functions may be applied to produce unique functionals, such as the delta measure. It is well known that the linear theory of generalized functions may be extended to a nonstandard theory of generalized functions, which reproduces the basic results of the standard theory, Laugwitz [21] and Richter [36]. In the standard theory the delta measure is a unique functional resulting from the abstract limits of regularizing sequences; on the other hand in 
the hyperreal setting, the functional whose standard part produces the delta measure can be represented by uncountably many (internal) hyperreal functions with distinct microstructures. Such internal functions are called pre-distributions, Palmgren [37]. The significance of the nonstandard delta measures is in their utility; differentiable representations of such generalized functions may be written down explicitly and manipulated like standard differentiable functions. The focus of the present work is to exploit smooth representations of nonstandard generalized functions to derive shock wave jump conditions. No attempt is made in the present report to outline a nonstandard development of the linear theory of distributions.

The analysis of shock wave jump conditions requires the use of hyperreal Heaviside functions. Like the delta measure, the Heaviside function can be represented by uncountably many differentiable internal functions with distinct microstructures. To introduce a nonstandard Heaviside function, let $\varepsilon_{1}$ and $\varepsilon_{2}$ be two infinitesimal numbers where at least one of them is not zero, and where $\varepsilon_{1} \approx \varepsilon_{2}$ and $\varepsilon_{1}<\varepsilon_{2}$. An internal Heaviside function $H(x)$ is defined as follows:

$$
H(x)=\left\{\begin{array}{cc}
0 & \text { if } x \leq \varepsilon_{1} \\
h(x) & \text { if } \varepsilon_{1}<x<\varepsilon_{2} \\
1 & \text { if } \quad x \geq \varepsilon_{2}
\end{array},\right.
$$

where

$$
\begin{gathered}
0=* 0=\{0,0,0,0, \ldots\}, \\
h(x)=* h(* x)=\left\{\left(h_{n}\left(x_{n}\right)\right)\right\},
\end{gathered}
$$

and

$$
1=* 1=\{1,1,1,1, \ldots\} .
$$

(Here and in the following development the *-notation is suppressed.) Moreover, $h(x)$ is assumed to be a differentiable function, not necessarily monotonically increasing, which satisfies

$$
h\left(\varepsilon_{1}\right)=0 \text { and } h\left(\varepsilon_{2}\right)=1 .
$$

As an example, the following function can be used for $h(x)$ when $x$ is in the open infinitesimal interval $\left(\varepsilon_{1}, \varepsilon_{2}\right)$.

$$
h(x)=\frac{1}{2}-\frac{1}{2} \cos \left[\frac{\pi\left(\varepsilon_{2}-x\right)}{\varepsilon_{2}-\varepsilon_{1}}\right] .
$$


Example 20. Figure 1 shows the standard Heaviside function

$$
H_{\mathrm{STD}}(x)=\left\{\begin{array}{lll}
0 & \text { if } & x<0 \\
1 & \text { if } & x>0
\end{array},\right.
$$

and the nonstandard Heaviside $H(x)$ function given by combining equations (3.13) and (3.11):

$$
H(x)=\left\{\begin{array}{cll}
0 & \text { if } & x \leq 0 \\
\frac{1}{2}-\frac{1}{2} \cos \left[\frac{\pi x}{\varepsilon}\right] & \text { if } 0<x<\varepsilon, \\
1 & \text { if } & x \geq \varepsilon
\end{array}\right.
$$

where $\varepsilon$ is a positive infinitesimal. All the plots of the hyperreal functions are normalized on the finite interval $[0,1]$ by setting $x \rightarrow x / \varepsilon$. Notice that the standard Heaviside function has a jump at $x=0$ but the nonstandard Heaviside function is continuous on $[0, \varepsilon]$. Also notice that $H(x)$ is a differentiable function on $(0, \varepsilon)$ since $h(x)$ is differentiable. The derivative of $H(x)$ is the internal function

$$
H^{\prime}(x)=\left\{\begin{array}{c}
0 \quad \text { if } \quad x \leq 0 \\
\frac{\pi}{2 \varepsilon} \sin \left[\frac{\pi x}{\varepsilon}\right] \quad \text { if } 0<x<\varepsilon, \\
0 \quad \text { if } \quad x \geq \varepsilon
\end{array}\right.
$$

which is a pre-distribution for the Dirac delta. For all real test functions $\phi \in D(\mathbf{R})$ it may be shown that

$$
\operatorname{st}\left(\int_{-\infty}^{\infty} H^{\prime}(x) \cdot \phi(x) d x\right)=\phi(0)
$$

following a calculation similar to that performed in Example 19. An important property of $H^{\prime}(x)$ is

$$
\int_{-\infty}^{\infty} H^{\prime}(x) d x=\frac{\Omega \pi}{2} \cdot \int_{0}^{\varepsilon(\Omega)} \sin [\pi \Omega x] d x=1,
$$


where $\Omega$ is any infinite number and $\varepsilon=\varepsilon(\Omega)$ is any infinitesimal number such that $\Omega \varepsilon=1$. Figure 2 shows the derivative of the nonstandard Heaviside function $H^{\prime}(x)$ of equation (3.14) on the normalized interval $[0,1]$.

Another important property is that the support of $H^{\prime}(x)$ is the closed infinitesimal interval $[0, \varepsilon]$. Suppose $\psi(x)$ is a standard function which is discontinuous at $x=0$ with right and left limits $\psi(0+)$ and $\psi(0-)$ respectively. Then because $\varepsilon>0$, the integral satisfies

$$
\mathrm{st}\left(\int_{-\infty}^{\infty} H^{\prime}(x) \cdot \psi(x) d x\right)=\psi(0+) .
$$

This means that for a function such as $\psi(x)$ with discontinuity at $x=0$, the derivative of $H(x)$ picks up $\psi(0+)$. By selecting $\varepsilon_{1}$ and $\varepsilon_{2}$ in equation (3.11), another nonstandard Heaviside function $G(x)$ may be found such that $G^{\prime}(x)$ will have the following property

$$
\mathrm{st}\left(\int_{-\infty}^{\infty} G^{\prime}(x) \cdot \psi(x) d x\right)=\alpha \psi(0-)+(1-\alpha) \psi(0+)
$$

where $0<\alpha<1$.

In particular, let $\varepsilon$ be a positive infinitesimal and $\varepsilon_{1}=-\frac{\varepsilon}{2}, \varepsilon_{2}=\frac{\varepsilon}{2}$ in equations (3.11) and (3.12) to define a differentiable Heaviside function:

$$
K(x)=\left\{\begin{array}{cc}
0 \quad \text { if } \quad x \leq-\frac{\varepsilon}{2} \\
\frac{1}{2}-\frac{1}{2} \cos \left[\frac{\pi(\varepsilon / 2+x)}{\varepsilon}\right] \quad \text { if }-\frac{\varepsilon}{2}<x<\frac{\varepsilon}{2}, \\
1 \quad \text { if } x \geq \frac{\varepsilon}{2}
\end{array}\right.
$$

Then $K^{\prime}(x)$ has the following property

$$
\mathrm{st}\left(\int_{-\infty}^{\infty} K^{\prime}(x) \cdot \psi(x) d x\right)=\frac{1}{2}(\psi(0-)+\psi(0+)) .
$$




\subsection{Multiplication of Nonstandard Generalized Functions}

The Schwartz theorem showing that generalized functions cannot be multiplied is a manifestation of a basic structural limitation of algebraic mathematics. Following Rosinger [5], consider the mathematical structure $\left(\mathfrak{I},{ }^{\circ}, D\right)$ consisting of a collection $\mathfrak{I}$ of functions defined on $\mathbf{R}$ which may exhibit a finite number of localized discontinuities. The standard Heaviside function (with the STD notation suppressed)

$$
H(x)=\left\{\begin{array}{lll}
0 & \text { if } & x<0 \\
1 & \text { if } & x>0
\end{array},\right.
$$

is contained in $\mathfrak{I}$. Let $\circ$ and $D$ denote the standard multiplication and differentiation operators acting on the set $\mathfrak{I}$.

From equation (3.15), notice that

$$
H^{m}(x)=(H \circ H \circ H \circ \ldots \circ H)(x)=H(x),
$$

for all $m>1$. Then applying the derivative operator in the sense of generalized functions to equation (3.16) yields

$$
m H^{m-1}(x) D H(x)=D H(x),
$$

which implies that

$$
m H^{m-1}(x)=1
$$

and

$$
H(x)=\frac{1}{m},
$$

for all $m>1$. But equation (3.17) contradicts the definition of the standard Heaviside function (3.15). Therefore, the mathematical structure $(\mathfrak{I}, \circ, D)$ is over-specified and discontinuity, multiplication and differentiation cannot be combined in the algebraic steps of a single derivation. The over-specification of $(\mathfrak{I}, \circ, D)$ may be repaired by requiring that

$$
H^{2}(x)=(H \circ H)(x) \neq H(x) .
$$

Recall that a hyperreal number system is a field; hence, hyperreal numbers may be multiplied. This implies that the hyperreal functions may be multiplied on intervals where they are defined. 
Note that for any Heaviside function defined by equation (3.11),

$$
H^{2}(x)=\left\{\begin{array}{cc}
0 & \text { if } \quad x \leq \varepsilon_{1} \\
h^{2}(x) & \text { if } \varepsilon_{1}<x<\varepsilon_{2} \\
1 & \text { if } \quad x \geq \varepsilon_{2}
\end{array}\right.
$$

which implies that

$$
H^{2}(x) \neq H(x)
$$

because $h^{2}(x) \neq h(x)$ for $\varepsilon_{1}<x<\varepsilon_{2}$. That is, giving the Heaviside function $H(x)$ a fixed microstructure removes the algebraic over-specification which leads to the contradiction of equation (3.17). Also note, that by taking derivative of both sides of equation (3.18)

$$
H(x) H^{\prime}(x)=\left\{\begin{array}{ccc}
0 & \text { if } & x \leq \varepsilon_{1} \\
h(x) h^{\prime}(x) & \text { if } & \varepsilon_{1}<x<\varepsilon_{2} . \\
0 & \text { if } & x \geq \varepsilon_{2}
\end{array}\right.
$$

For example, equations (3.11) and (3.13) yield

$$
H(x) H^{\prime}(x)=\left\{\begin{array}{c}
0 \quad \text { if } \quad x \leq 0 \\
\frac{\pi}{4 \varepsilon} \sin \left[\frac{\pi x}{\varepsilon}\right]\left(1-\cos \left[\frac{\pi x}{\varepsilon}\right]\right) \quad \text { if } 0<x<\varepsilon . \\
0 \quad \text { if } \quad x \geq \varepsilon
\end{array}\right.
$$

Therefore, $H(x) H^{\prime}(x)$ has a clearly defined hyperreal range assuming $\varepsilon$ is a given nonzero infinitesimal. Equation (3.11) generalizes for all differentiable nonstandard Heaviside functions to the result that

$$
H^{m}(x) \neq H(x),
$$

for all $m>1$, removing the contradictions obtained by setting

$$
H^{m}(x)=H(x)
$$

Example 21. Although the product of two smooth pre-distributions exists and is a smooth nonstandard function, such a product will not yield a standard distribution. To see this, recall the 
internal function of equation (3.9) representing the delta measure and then consider the functional acting on any standard test function $\phi \in D(\mathbf{R})$;

$$
\int_{-\infty}^{\infty} \delta(x) \cdot \delta(x) \cdot \phi(x) d x=\int_{-\infty}^{\infty}\left[\frac{1}{\pi} \cdot \frac{\Omega}{1+\Omega^{2} x^{2}}\right]^{2} \cdot \phi(x) d x
$$

where $\Omega$ is any infinite hyperreal and $\varepsilon=\varepsilon(\Omega)$ is any infinitesimal hyperreal such that $\Omega \varepsilon$ is an infinite hyperreal. Performing the integration yields

$$
\int_{-\infty}^{\infty}\left[\frac{1}{\pi} \cdot \frac{\Omega}{1+\Omega^{2} x^{2}}\right]^{2} \cdot \phi(x) d x \approx \frac{\phi(0)}{\pi^{2}} \cdot \int_{-\varepsilon(\Omega)}^{\varepsilon(\Omega)}\left[\frac{\Omega}{1+\Omega^{2} x^{2}}\right]^{2} d x
$$

where

$$
\left.\int_{-\infty}^{\infty}\left[\frac{1}{\pi} \cdot \frac{\Omega}{1+\Omega^{2} x^{2}}\right]^{2} \cdot \phi(x) d x \approx \frac{\phi(0)}{\pi^{2}} \cdot \Omega^{2}\left(\frac{1}{2} \cdot \frac{x}{1+\Omega^{2} x^{2}}+\frac{1}{2 \Omega} \cdot \arctan (\Omega x)\right)\right|_{-\varepsilon(\Omega)} ^{\varepsilon(\Omega)} .
$$

Then evaluating the limits of the integral

$$
\left.\frac{\phi(0)}{\pi^{2}} \cdot \Omega^{2}\left(\frac{1}{2} \cdot \frac{x}{1+\Omega^{2} x^{2}}+\frac{1}{2 \Omega} \cdot \arctan (\Omega x)\right)\right|_{-\varepsilon(\Omega)} ^{\varepsilon(\Omega)} \approx \frac{\phi(0)}{\pi^{2}} \cdot \Omega^{2} \cdot\left(\frac{\varepsilon}{1+\Omega^{2} \varepsilon^{2}}+\frac{\pi}{2 \Omega}\right) .
$$

Notice that the first term of the right-hand-side of the integral satisfies

$$
\frac{\phi(0)}{\pi^{2}} \cdot \Omega \cdot\left(\frac{\Omega \varepsilon}{1+\Omega^{2} \varepsilon^{2}}\right)=\frac{\phi(0)}{\pi^{2}} \cdot \frac{\Omega}{\frac{1}{\Omega \varepsilon}+\Omega \varepsilon} \approx \frac{\phi(0)}{\pi^{2}} \cdot \frac{1}{\varepsilon}
$$

while the second term may be written

$$
\frac{\phi(0)}{\pi^{2}} \cdot \Omega^{2} \cdot\left(\frac{\pi}{2 \Omega}\right)=\frac{\phi(0)}{2 \pi} \cdot \Omega .
$$

Therefore, the functional for the square of delta measure with the pre-distribution of (3.9) produces

$$
\int_{-\infty}^{\infty} \delta(x) \cdot \delta(x) \cdot \phi(x) d x=\int_{-\infty}^{\infty}\left[\frac{1}{\pi} \cdot \frac{\Omega}{1+\Omega^{2} x^{2}}\right]^{2} \cdot \phi(x) d x \approx \phi(0) \cdot\left(\frac{1}{\pi^{2}} \cdot \frac{1}{\varepsilon}+\frac{1}{2 \pi} \cdot \Omega\right),
$$

which is an infinite hyperreal number. And since the integral of the square of the Dirac delta is an infinite number, the standard part of the integral is infinite. Hence, the square of the delta measure 
does not yield a generalized function; and, the specific hyperreal number produced for $\delta \cdot \delta$ depends on the choice of the pre-distribution used to represent the delta measure.

Example 22. The result of equation (3.20) and its interpretation for the square of the delta measure changes dramatically if the functional of equation (3.19) is assumed to act on a space of nonstandard internal test functions $D(* \mathbf{R})$ instead of a space of standard test functions $D(\mathbf{R})$. To see this, choose any internal test function $\phi \in D\left({ }^{*} \mathbf{R}\right)$ such that

$$
\phi(0)=\varepsilon^{2},
$$

where $\Omega$ is any infinite hyperreal and $\varepsilon=\varepsilon(\Omega)$ is any infinitesimal hyperreal such that $\Omega \varepsilon$ is infinite and $\Omega \varepsilon^{2}$ is finite. Integrating equation (3.19) then yields

$$
\int_{-\infty}^{\infty} \delta(x) \cdot \delta(x) \cdot \phi(x) d x \approx \phi(0) \cdot\left(\frac{1}{\pi^{2}} \cdot \frac{1}{\varepsilon}+\frac{1}{2 \pi} \cdot \Omega\right) \approx \frac{\varepsilon}{\pi^{2}}+\frac{1}{2 \pi} \cdot \Omega \cdot \varepsilon^{2},
$$

and taking the standard part of the integral produces the finite value:

$$
\mathrm{st}\left(\int_{-\infty}^{\infty} \delta(x) \cdot \delta(x) \cdot \phi(x) d x\right)=\frac{1}{2 \pi} \cdot \mathrm{st}\left(\Omega \cdot \varepsilon^{2}\right) .
$$

Hence, the square of the delta measure (3.21) yields a nonstandard object which is analogous to a Colombeau nonlinear generalized function. The specific finite number produced for $\delta \cdot \delta$ depends on the choice of the pre-distribution used to represent the delta measure as well as the choice of the nonstandard test function.

Example 23. As a second example of a product of generalized functions, consider $\delta \cdot H$. The multiplication of the delta measure with the Heaviside function occurs in the analysis and computation of shock waves if the governing equations of motion are written in nonconservative form; $\delta \cdot H$ comes from the nonlinear terms, such as the convective acceleration term $u u_{x}$, for a discontinuity in the field variable, $u$. In the standard theory of generalized functions, the product $\delta \cdot H$ does not yield a well defined functional, because the results depend on the representations 
of $\delta$ and $H$. To see this behavior, choose the following pre-distributions for the delta measure and the Heaviside function:

$$
\begin{gathered}
\delta(x)=\frac{2}{\pi} \cdot \frac{\Omega}{\exp (\Omega x)+\exp (-\Omega x)}, \\
H(x)=\left\{\begin{array}{ccc}
0 & \text { if } & x \leq 0 \\
1-\exp (-\Omega x) & \text { if } & 0<x<\varepsilon, \\
1 & \text { if } & x \geq \varepsilon
\end{array}\right.
\end{gathered}
$$

where $\Omega$ is any infinite hyperreal and $\varepsilon=\varepsilon(\Omega)$ is any infinitesimal hyperreal such that $\Omega \varepsilon$ is an infinite hyperreal. Now, consider the functional acting on any standard test function $\phi \in D(\mathbf{R})$ :

$$
\int_{-\infty}^{\infty} \delta(x) \cdot H(x) \cdot \phi(x) d x=\int_{0}^{\infty} \frac{2}{\pi} \cdot \frac{\Omega(1-\exp (-\Omega x))}{\exp (\Omega x)+\exp (-\Omega x)} \cdot \phi(x) d x .
$$

Integrating equation (3.22) yields

$$
\int_{0}^{\infty} \frac{2}{\pi} \cdot \frac{\Omega(1-\exp (-\Omega x))}{\exp (\Omega x)+\exp (-\Omega x)} \cdot \phi(x) d x \approx 2 \phi(0) \frac{\Omega}{\pi} \int_{0}^{\varepsilon(\Omega)} \frac{\Omega(1-\exp (-\Omega x))}{\exp (\Omega x)+\exp (-\Omega x)} d x
$$

where

$$
\begin{aligned}
& \int_{0}^{\infty} \frac{2}{\pi} \cdot \frac{\Omega(1-\exp (-\Omega x))}{\exp (\Omega x)+\exp (-\Omega x)} \cdot \phi(x) d x \approx \\
& \left.\quad \frac{\phi(0)}{\pi}\{2 \arctan ((\exp (\Omega x))+\ln (1+\exp (2 \Omega x))-2 \Omega x)\}\right|_{0} ^{\varepsilon(\Omega)} \approx \phi(0)\left\{\frac{1}{2}-\frac{\ln 2}{\pi}\right\} .
\end{aligned}
$$

Hence,

$$
\mathrm{st}\left(\int_{-\infty}^{\infty} \delta(x) \cdot H(x) \cdot \phi(x) d x\right) \cong 0.27936 \cdot \phi(0)
$$

If other pre-distributions are selected for $\delta$ and $H$, the numerical value multiplying $\phi(0)$ in equation (3.21) will, in general, be different. In the numerical approximation of shock problems, the use of nonconservative forms of the equations of motion produce shock wave speeds that depend on the numerical scheme and not the physics of shock propagation. Nonconservative 
numerical simulations of one-dimensional shock problems may be found in Baty [38]. Such numerical approximations are analogous to the result of equation (3.21); the specific numerical values are fixed by the choice of the pre-distributions for $\delta$ and $H$.

Example 24. Again, consider the product $\delta \cdot H$, but now assume $\delta=H^{\prime}$. Then for any predistribution representing the delta measure, $H^{\prime}$ defined on the interval $(0, \varepsilon)$, choose hyperreal numbers $\Omega, \varepsilon=\varepsilon(\Omega)$ so that $\Omega$ is infinite, $\varepsilon$ is infinitesimal and $\Omega \varepsilon$ is an infinite hyperreal. Then for any standard test function $\phi \in D(\mathbf{R})$, the functional equation for $\delta \cdot H$ implies:

$$
\int_{-\infty}^{\infty} \delta(x) \cdot H(x) \cdot \phi(x) d x=\int_{-\infty}^{\infty} H^{\prime}(x) \cdot H(x) \cdot \phi(x) d x
$$

and,

$$
\int_{-\infty}^{\infty} \delta(x) \cdot H(x) \cdot \phi(x) d x=\int_{-\infty}^{\infty} \frac{d}{d x}\left(\frac{1}{2} \cdot H^{2}(x)\right) \cdot \phi(x) d x \approx \phi(0) \int_{0}^{\varepsilon(\Omega)} \frac{d}{d x}\left(\frac{1}{2} \cdot H^{2}(x)\right) d x
$$

Therefore,

$$
\mathrm{st}\left(\int_{-\infty}^{\infty} \delta(x) \cdot H(x) \cdot \phi(x) d x\right)=\frac{1}{2} \cdot \phi(0)
$$

Equation (3.25) is consistent with the results obtained by the standard theory of generalized functions. This special case of the product $\delta \cdot H$ may be computed using only discontinuity and multiplication and is not over-specified, since $\delta \cdot H$ is not differentiated. The differentiation of the Heaviside function is avoided by applying integration by parts. To this end, consider the standard version of equation (3.24):

$$
\int_{-\infty}^{\infty} \delta(x) \cdot H(x) \cdot \phi(x) d x=\int_{-\infty}^{\infty} \frac{d}{d x}\left(\frac{1}{2} \cdot H^{2}(x)\right) \cdot \phi(x) d x=\int_{-\infty}^{\infty} \frac{d}{d x}\left(\frac{1}{2} \cdot H(x)\right) \phi(x) d x
$$

which assumes that $H(x)=H^{2}(x)$. Equation (3.24) then implies: 


$$
\int_{-\infty}^{\infty} \frac{d}{d x}\left(\frac{1}{2} \cdot H(x)\right) \phi(x) d x=-\frac{1}{2} \cdot \int_{0}^{\infty} \frac{d}{d x} \phi(x) d x=\frac{1}{2} \cdot \phi(0)
$$

producing the same result as equation (3.25). This example is analogous to integrating the governing equations of motion modeling shock wave propagation in conservative form: the predicted shock wave speed will be independent of the numerical approximation.

Notice that the standard calculation performed in Example 24 to reproduce the result of equation (3.25) depends on the order of the algebraic and analytic steps. Changing the order of the operations can produce an over-specified problem with ambiguous results. This demonstrates the power of applying nonstandard analysis to problems involving products of general functions: the mathematical inconsistencies are removed and the problem may often be reduced to elementary calculus.

\subsection{INVISCID SHOCK WAVE JUMP CONDITIONS}

\subsection{Specific Volume, Velocity and Pressure Jump Conditions}

Consider the motion of a normal shock wave propagating through a quiescent, perfect gas. On either side of the shock wave the specific volume $v$, velocity $u$, pressure $p$, and entropy $s$, of the flow are assumed to be constant. The goal of this section is to derive the jump conditions for specific volume, velocity and pressure across a normal shock assuming that the governing equations of motion are expressed in nonconservative form. The jump conditions for specific volume and pressure are then applied in the following section to derive the jump condition for entropy. Since the values of the field variables are constant on either side of the shock wave, the shock wave does not accelerate, so that the shock speed is constant. Hence, the characteristics for the shock wave are straight lines in the space-time domain. Along the characteristic lines the governing equations reduce to total or ordinary differential equations, so the field variables $v, u$, and $p$, may be assumed to have the following form of equation (1.2) across the shock wave:

$$
\begin{aligned}
& v(\xi)=v_{l}+[v] H(\xi), \\
& u(\xi)=u_{l}+[u] K(\xi),
\end{aligned}
$$




$$
p(\xi)=p_{l}+[p] L(\xi),
$$

where $\xi=x+c t$ is a characteristic line, $c$ is the shock speed, and where $[\phi] \equiv \phi_{r}-\phi_{l}$, with the subscripts $r$ and $l$ referring to the right (downstream) and left (upstream) conditions across the shock, respectively. The functions $H(\xi), K(\xi)$, and $L(\xi)$ are assumed to be smooth (internal) nonstandard Heaviside functions as described in Chapter 3. Each one of these Heaviside functions is taken to have its jump contained on the same infinitesimal interval $(0, \varepsilon)$.

Now substituting equations (4.1) and (4.2) into the continuity equation (2.17) yields:

$$
c[v] H^{\prime}-\left(v_{l}+[v] H\right)[u] K^{\prime}+\left(u_{l}+[u] K\right)[v] H^{\prime}=0,
$$

where $X^{\prime} \equiv \frac{d X}{d \xi}$. Defining $\tilde{u}=u+c$ and collecting terms in equation (4.4) produces

$$
\left(\tilde{u}_{l}+[u] K\right)[v] H^{\prime}-\left(v_{l}+[v] H\right)[u] K^{\prime}=0,
$$

which implies:

$$
\frac{[v] H^{\prime}}{v_{l}+[v] H}=\frac{[u] K^{\prime}}{\tilde{u}_{l}+[u] K} .
$$

Integrating both sides of equation of (4.5) then gives:

$$
H=-\frac{v_{l}}{[v]}+\alpha\left(\frac{\tilde{u}_{l}}{[u]}+K\right),
$$

where $\alpha$ depends on the constant of integration. Applying the fact that $H, K=0$ for $\xi=0$, yields:

$$
\alpha=\frac{v_{l}[u]}{[v] \tilde{u}_{l}}
$$

and hence,

$$
H=\frac{v_{l}[u]}{[v] \tilde{u}_{l}} K
$$

Recalling that $H, K=1$ for $\xi=\varepsilon$, then implies:

$$
\frac{v_{l}[u]}{[v] \tilde{u}_{l}}=1 .
$$

And combining equations (4.6) and (4.7) yields,

$$
H=K,
$$


so that $H$ and $K$ are the same function defined on the infinitesimal interval $(0, \varepsilon)$, i.e., $H$ and $K$ have the same microstructure. Notice that equation (4.7) is equivalent to the classical shock wave jump condition for conservation of mass:

$$
\rho_{l} \tilde{u}_{l}=\rho_{r} \tilde{u}_{r} .
$$

Next, substituting equations (4.1), (4.2) and (4.3) into the momentum equation (2.18) yields:

$$
c[u] K^{\prime}+\left(u_{l}+[u] K\right)[u] K^{\prime}+\left(v_{l}+[v] H\right)[p] L^{\prime}=0 .
$$

Applying $H=K$ from equation (4.8) produces the separable equation:

$$
\frac{\left(\tilde{u}_{l}+[u] K\right)[u] K^{\prime}}{v_{l}+[v] K}+[p] L^{\prime}=0 .
$$

Then combining equations (4.7) and (4.10) implies:

$$
\frac{[u]^{2}}{[v]} K^{\prime}+[p] L^{\prime}=0 .
$$

Integrating equation (4.11) gives:

$$
L=-\frac{[u]^{2}}{[v][p]} K+\beta,
$$

where $\beta$ is a constant of integration. Applying the property that $L, K=0$ for $\xi=0$, gives $\beta=0$; while applying $L, K=1$ for $\xi=\varepsilon$, implies:

$$
\frac{[u]^{2}}{[v][p]}=-1 .
$$

Equation (4.13) is equivalent to the Prandtl relation:

$$
\tilde{u}_{r} \tilde{u}_{l}=[p][v]=\frac{p_{r}-p_{l}}{\rho_{r}-\rho_{l}} .
$$

Whence, equations (4.12) and (4.13) show that the microstructure for the jump functions $K$ and $L$ is the same:

$$
K=L .
$$

Finally, by combining equations (4.8) and (4.14) it follows that the microstructure for the Heaviside functions for the specific volume, velocity, and pressure jump conditions are coincident across an inviscid shock wave: 


$$
H=K=L .
$$

An important observation should be drawn from the analysis producing equation (4.15). The algebraic and analytic operations shown in equations (4.4) through (4.13) appear to follow closely well known calculations such as those performed by Colombeau and Le Roux, [10] and Salas and Iollo [18] yielding the same result. In these works, however, the objects being manipulated are nonlinear generalized functions (functional equations) written in a symbolic notation similar to that used in advanced calculus; these objects are not smooth functions and do not support all of the operations of ordinary algebra and calculus; the multiplication of singular generalized functions is accomplished via a weak equality called association. In contrast to such calculations, the objects manipulated in equations (4.4) to (4.13) (and indeed, in the following sections of this report) are smooth nonstandard functions. Therefore, the algebraic and analytic manipulations are the standard operations for smooth functions extended to smooth internal functions.

Example 25. Notice that the derivation of equation (4.15) by using the governing equations in nonconservative form requires manipulating products of the pre-distributions, $H(\xi), K(\xi)$, and $L(\xi)$ and their derivatives. Since these functions are differentiable the mathematical structures applied in this analysis are not over-specified and will produce the same results obtained by analyzing the problem in conservative form. For example, an expression for the shock speed $c$ may be derived using equation (4.7):

$$
\frac{v_{l}[u]}{[v] \tilde{u}_{l}}=1 .
$$

This relation implies

$$
\tilde{u}_{l}=\frac{v_{l}[u]}{[v]}
$$

and since $\tilde{u}_{l}=u_{l}+c$, it follows that

$$
c=\frac{1}{[v]}\left(v_{l}[u]-u_{l}[v]\right) .
$$

Equation (4.16) is the shock speed obtained from the equations of motion in conservative form (which is also the physically observed shock speed). 


\subsection{Entropy Jump Condition}

To derive the entropy jump condition, as in Section 4.1 assume a solution for the entropy $s$ in the form of equation (1.2) across the shock wave

$$
s(\xi)=s_{l}+[s] S(\xi),
$$

where $\xi=x+c t$ is a characteristic line, $c$ is the shock speed, and where $[s] \equiv s_{r}-s_{l}$, with the subscripts $r$ and $l$ referring to the right and left conditions across the shock, respectively. Combining equations (4.2), (4.3), (4.14), and (4.17) with the energy equation (2.15) yields:

$$
\begin{aligned}
& \left(p_{l}+[p] H\right)\left(c[s] S^{\prime}+\left(u_{l}+[u] H\right)[s] S^{\prime}\right)= \\
& c_{v}\left(c[p] H^{\prime}+\left(u_{l}+[u] H\right)[p] H^{\prime}+\gamma\left(p_{l}+[p] H\right)[u] H^{\prime}\right)
\end{aligned}
$$

which implies

$$
\left(p_{l}+[p] H\right)\left(\tilde{u}_{l}+[u] H\right)[s] S^{\prime}=c_{v}\left(\left(\tilde{u}_{l}+[u] H\right)[p] H^{\prime}+\gamma\left(p_{l}+[p] H\right)[u] H^{\prime}\right)
$$

Equation (4.18) then produces:

$$
[s] S^{\prime}=c_{v}\left\{\left(\frac{[p] H^{\prime}}{p_{l}+[p] H}\right)+\gamma\left(\frac{[u] H^{\prime}}{\tilde{u}_{l}+[u] H}\right)\right\} .
$$

Recalling that equation (4.7) gives

$$
\tilde{u}_{l}=\frac{v_{l}[u]}{[v]}
$$

implies that equation (4.19) may be written:

$$
[s] S^{\prime}=c_{v}\left\{\left(\frac{[p] H^{\prime}}{p_{l}+[p] H}\right)+\gamma\left(\frac{[v] H^{\prime}}{v_{l}+[v] H}\right)\right\} .
$$

Integrating equation (4.20) then produces:

$$
[s] S=c_{v} \log \left(\left(p_{l}+[p] H\right) \cdot\left(v_{l}+[v] H\right)^{\gamma}\right)+\zeta,
$$

where $\zeta$ is a constant of integration. Now, applying the property that $H=0$ for $\xi=0$, gives

$$
\zeta=-c_{v} \log \left(p_{l} v_{l}^{\gamma}\right)
$$

while applying $H=1$ for $\xi=\varepsilon$, yields,

$$
[s]=c_{v} \log \left(\left(1+\frac{[p]}{p_{l}}\right) \cdot\left(1+\frac{[v]}{v_{l}}\right)^{\gamma}\right) .
$$


Equations (4.21), (4.22), and (4.23) then yield the jump function for entropy across an inviscid shock wave:

$$
S=\frac{c_{v}}{[s]} \log \left(\left(1+\frac{[p]}{p_{l}} H\right) \cdot\left(1+\frac{[v]}{v_{l}} H\right)^{\gamma}\right)
$$

Notice that equation (4.24) may be derived from the thermodynamic equation for the entropy, equation (2.19):

$$
s=s_{l}+[s] S=c_{v} \log \left(\left(p_{l}+[p] H\right) \cdot\left(v_{l}+[v] H\right)^{\gamma}\right) .
$$

Hence,

$$
s=c_{v} \log \left(p_{l} v_{l}^{\gamma}\right)+c_{v} \log \left(\left(1+\frac{[p]}{p_{l}} H\right) \cdot\left(1+\frac{[v]}{v_{l}} H\right)^{\gamma}\right)
$$

where

$$
s_{l}=c_{v} \log \left(p_{l} v_{l}^{\gamma}\right)
$$

Equation (4.25) shows that the jump function for entropy is a nonlinear function of $H$. If the Heaviside function $H$ is assumed to be smooth and strictly monotonically increasing on the infinitesimal interval $(0, \varepsilon)$, then the entropy jump function exhibits a peak in the shock layer: $s$ increases from $s_{l}$ at 0 to a peak and then decreases to $s_{r}$ at $\varepsilon$ such that $s_{l}<s_{r}$. To see this, differentiate equation (4.25) to produce:

$$
\frac{d s}{d \xi}=c_{v} \cdot \frac{\left([p] / p_{l}\right) H^{\prime}}{1+\left([p] / p_{l}\right) H}+c_{v} \cdot \frac{\gamma\left([v] / v_{l}\right) H^{\prime}}{1+\left([v] / v_{l}\right) H}
$$

To find an extreme point, set

$$
\frac{d s}{d \xi}=0
$$

Since $H$ is a smooth, strictly monotonically increasing function, equations (4.26) and (4.27) give

$$
\frac{[p]}{p_{l}}\left(1+\left([v] / v_{l}\right) H\right)+\gamma \frac{[v]}{v_{l}}\left(1+\left([p] / p_{l}\right) H\right)=0
$$

which implies

$$
\frac{[p]}{p_{l}}+\gamma \frac{[v]}{v_{l}}+(\gamma+1) \frac{[p]}{p_{l}} \frac{[v]}{v_{l}} H\left(\xi_{p}\right)=0 .
$$


Recall from gas dynamics that pressure and specific volume jump terms may be related through the Rankine-Hugoniot equation for a perfect gas:

$$
\frac{[v]}{v_{l}}=\frac{-2[p] / p_{l}}{2 \gamma+(\gamma+1)[p] / p_{l}},
$$

Thompson [23]. Now, combining equations (4.28) and (4.29) produces:

$$
\frac{[p]}{p_{l}}+\gamma\left(\frac{-2[p] / p_{l}}{2 \gamma+(\gamma+1)[p] / p_{l}}\right)+(\gamma+1) \frac{[p]}{p_{l}}\left(\frac{-2[p] / p_{l}}{2 \gamma+(\gamma+1)[p] / p_{l}}\right) H\left(\xi_{p}\right)=0,
$$

which reduces to

$$
2 \gamma+(\gamma+1) \frac{[p]}{p_{l}}-2 \gamma-2(\gamma+1) \frac{[p]}{p_{l}} H\left(\xi_{p}\right)=0,
$$

and hence,

$$
H\left(\xi_{p}\right)=\frac{1}{2}
$$

And from the assumptions on $H$, a point $\xi_{p} \in(0, \varepsilon)$ exists statisfying equation (4.30). This derivation yields the following result:

Theorem 26. If $H$ is a smooth, strictly monotonically increasing, internal Heaviside function, the entropy jump function of equation (4.25) admits a maximum value in the shock layer $(0, \varepsilon)$.

From both theoretical and experimental work, it is known that $H$ is a strictly monotonically increasing function, Muntz and Harnett [39]. Furthermore, the existence of the entropy peak in the shock layer is well known and has been derived using a viscous flow assumption, Morduchow and Libby [17] and using Colombeau's nonlinear generalized functions, Salas and Iollo [18]. The present analysis demonstrates that the entropy peak is caused by combining a nonlinear expression with a jump condition and does not depend on the viscosity of the fluid.

To plot examples of the normalized entropy jump in the shock layer, the smooth internal Heaviside function is assumed for the specific volume: 


$$
H(\xi)=\frac{1}{2}+\frac{1}{\pi} \cdot \arctan \left(\Omega\left\{\xi-\frac{\varepsilon}{2}\right\}\right),
$$

where $\Omega$ is an infinite hyperreal and $\varepsilon$ is an infinitesimal hyperreal such that $\Omega \varepsilon$ is an infinite hyperreal. On the interval $[0, \varepsilon]$ the Heaviside function of equation (4.31) satisfies:

$$
H(0) \approx 0 \text { and } H(\varepsilon) \approx 1 \text {. }
$$

Figure 3 shows equation (4.31) on the normalized jump interval of $[0,1]$ with $\Omega=100$. Now, if the pressure and specific volume jump terms [ ], of equation (4.24) are written as functions of the Mach number, $M$ :

$$
\frac{[p]}{p_{l}}=\frac{2 \gamma}{\gamma+1}\left(M^{2}-1\right)
$$

and

$$
\frac{[v]}{v_{l}}=-\frac{2}{\gamma+1}\left(1-\frac{1}{M^{2}}\right)
$$

with the upstream flow variables set to $p_{l}=1$ and $v_{l}=1$; the entropy jump function may be plotted. Figure 4 shows the variation of $S(\xi / \varepsilon)$ on the normalized jump interval [0,1] for a perfect gas flow with $\gamma=\frac{7}{5}$ for the normal shock wave Mach numbers $M=1.5,2.0,2.5,3.0$.

These entropy jump functions shown in Figure 4 exhibit peak values as required by Theorem 26. Notice that the peaks all occur at $\xi / \varepsilon=\frac{1}{2}$. This is the result of the choice of the Heaviside function in equation (4.31). As shown in the proof of Theorem 26, the peak of the entropy occurs where the jump function obtains the value $H\left(\xi_{p}\right)=\frac{1}{2}$. Since,

$$
H(1 / 2)=\frac{1}{2}
$$

the peak occurs at the mid point in the jump interval. Recall that the inviscid analysis does not determine the specific Heaviside function for the field variables; only the relationship between the field variable jump functions is found. Hence, different representations of the jump function for specific volume will yield different entropy shapes and entropy peak locations. The key point of 
the theorem is that a peak must occur for any strictly monotonically increasing jump function assumed for the specific volume.

Example 27. An analysis similar to that used to derive the entropy jump condition shows that across the shock wave:

$$
p\left(s_{t}+u s_{x}\right) \neq 0 .
$$

Suppose that equation (4.34) is zero across a shock wave. Recall from Chapter 2, that

$$
p\left(s_{t}+u s_{x}\right)=c_{v}\left(p_{t}+u p_{x}+\gamma p u_{x}\right)
$$

so that

$$
p\left(s_{t}+u s_{x}\right)=0
$$

implies:

$$
p_{t}+u p_{x}+\gamma p u_{x}=0 .
$$

Substituting equations (4.2) and (4.3) into equation (4.35) and applying equation (4.17) produces,

$$
\left(\left(\tilde{u}_{l}+[u] H\right)[p]+\gamma\left(p_{l}+[p] H\right)[u]\right) H^{\prime}=0,
$$

which may be written:

$$
\left(\tilde{u}_{l}[p]+\gamma p_{l}[u]+(\gamma+1)[u][p] H\right) H^{\prime}=0 .
$$

Equation (4.36) implies that either

$$
H^{\prime}=0 \text {, }
$$

or

$$
\tilde{u}_{l}[p]+\gamma p_{l}[u]+(\gamma+1)[u][p] H=0,
$$

must hold. Equation (4.37) cannot be hold because $H$ is not constant; hence, equation (4.38) must be considered. By applying the values on the ends of the jump interval, $\xi=0$ and $\xi=\varepsilon$, to $H$, equation (4.38) yields two expressions:

$$
\tilde{u}_{l}[p]+\gamma p_{l}[u]=0
$$

and

$$
\tilde{u}_{l}[p]+\gamma p_{l}[u]+(\gamma+1)[u][p]=0 .
$$

Then combining equation (4.39) with equation (4.40) produces:

$$
[u][p]=0 .
$$


Equation (4.41) implies that either the velocity or the pressure is constant across the shock wave, which is physically incorrect (a basic contradiction). The upshot is that none of the inviscid shock wave jump conditions may be derived in an unambiguous way by assuming

$$
s_{t}+u s_{x}=0 \text {. }
$$

\subsection{VISCOUS SHOCK WAVE JUMP CONDITIONS}

\subsection{Jump Conditions for a Viscous Non-Heat Conducting Gas}

Consider the motion of a normal shock wave propagating through a quiescent, viscous, perfect gas. For the analysis of this chapter, the notation defined in Section 4.1 will be applied. The goal of this section is to derive the jump conditions for specific volume, velocity, pressure, and entropy across a normal shock assuming that the governing equations of motion are expressed in nonconservative form. For the present analysis the coefficients of visocity $\mu$ and thermal conductivity $k$ are assumed to be constant. To derive these jump conditions, assume solutions for the field variables $v, u, p$, and $s$ in the form of equation (1.2) across the shock wave:

$$
\begin{gathered}
v(\xi)=v_{l}+[v] H(\xi), \\
u(\xi)=u_{l}+[u] K(\xi), \\
p(\xi)=p_{l}+[p] L(\xi), \\
s(\xi)=s_{l}+[s] S(\xi) .
\end{gathered}
$$

The functions $H(\xi), K(\xi), L(\xi)$, and $S(\xi)$ are smooth nonstandard Heaviside functions with their jumps contained on the infinitesimal interval $(0, \varepsilon)$.

Applying equations (5.1) and (5.2) with the continuity equation (2.26), a relationship between the Heaviside functions for specific volume and velocity may be determined. This analysis is identical to the inviscid derivation shown in equations (4.4) through (4.8) which produces:

$$
H=K \text {. }
$$

Hence, the specific volume and the velocity have the same microstructure across the shock wave. 
Next to develop a relationship between the Heaviside functions for the specific volume and the pressure, equations (5.1), (5.2) and (5.3) are substituted in the momentum equation (2.27):

$$
c[u] K^{\prime}+\left(u_{l}+[u] K\right)[u] K^{\prime}+\left(v_{l}+[v] H\right)[p] L^{\prime}-\frac{4}{3} \mu\left(v_{l}+[v] H\right)[u] K^{\prime \prime}=0 .
$$

Applying equation (5.5) to equation (5.6) produces:

$$
c[u] H^{\prime}+\left(u_{l}+[u] H\right)[u] H^{\prime}+\left(v_{l}+[v] H\right)[p] L^{\prime}-\frac{4}{3} \mu\left(v_{l}+[v] H\right)[u] H^{\prime \prime}=0,
$$

which implies,

$$
\frac{\left(u_{l}+c\right)[u]+[u]^{2} H}{v_{l}+[v] H} H^{\prime}+[p] L^{\prime}-\frac{4}{3} \mu[u] H^{\prime \prime}=0 .
$$

Combining $\tilde{u}_{l}=u_{l}+c$ with equations (4.7), and (5.7) yields:

$$
\frac{[u]^{2}}{[v]} H^{\prime}+[p] L^{\prime}-\frac{4}{3} \mu[u] H^{\prime \prime}=0,
$$

or

$$
H^{\prime \prime}-\frac{[u]}{\beta[v]} H^{\prime}-\frac{[p]}{\beta[u]} L^{\prime}=0 .
$$

where $\beta \equiv \frac{4}{3} \mu$. Integrating equation (5.8) with respect to the characteristic variable gives:

$$
H^{\prime}-\frac{[u]}{\beta[v]} H=\frac{[p]}{\beta[u]} L+\zeta,
$$

here $\zeta$ is a constant of integration. Applying the boundary conditions $H, H^{\prime}, K=0$ for $\xi=0$, shows that the constant of integration is zero:

$$
\zeta=0 .
$$

And applying the boundary conditions $H, K=1$ and $H^{\prime}=0$ for $\xi=\varepsilon$, then implies:

$$
[u]^{2}=-[v][p],
$$

which is the same condition found using the inviscid momentum equation, (4.13). Equations (5.9) to (5.11) give:

$$
H^{\prime}+\frac{[p]}{\beta[u]} H=\frac{[p]}{\beta[u]} L,
$$


which is a linear, non-homogeneous ordinary differential equation. Integrating (5.12) with respect to the characteristic variable $\xi$ and applying the boundary condition $H(0)=0$ then produces:

$$
H(\xi)=\Phi \int_{0}^{\xi} \exp (\Phi\{\varsigma-\xi\}) L(\varsigma) d \varsigma
$$

where $\Phi \equiv \frac{[p]}{\beta[u]}$, and hence establishing the relationship between $H$ and $L$.

Continuing, another equation relating the Heaviside functions $H$ and $L$ may be derived using the energy equation (2.30) for a viscous, heat conducting, perfect gas. Recall that:

$$
p v=R T \text {. }
$$

Notice that assuming the field variables satisfy the functional form (1.2) it follows that:

$$
(p v)_{t}=c(p v)_{x}
$$

Then combining equations (5.14) and (5.15) with equation (2.30) implies

$$
\frac{1}{\gamma-1} \tilde{u}(p v)_{x}+v p u_{x}-\eta v(p v)_{x x}-\beta v\left(u_{x}\right)^{2}=0
$$

where $\eta=\frac{k}{R}$. From equations (5.1), (5.2), (5.5), and (4.7) it follows that

$$
\tilde{u}=\frac{\tilde{u}_{l}}{v_{l}} v
$$

From (5.17) equation (5.16) then becomes

$$
\alpha(p v)_{x}+p u_{x}-\eta(p v)_{x x}-\beta\left(u_{x}\right)^{2}=0,
$$

and hence,

$$
\alpha\left(p_{x} v+p v_{x}\right)+p u_{x}-\eta\left(p_{x x} v+2 p_{x} v_{x}+p v_{x x}\right)-\beta\left(u_{x}\right)^{2}=0
$$

where $\alpha=\frac{1}{\gamma-1} \cdot \frac{\tilde{u}_{l}}{v_{l}}$.

Next, applying equations (5.1), (5.2), and (5.3) with $H=K$ and to equation (5.18) then yields

$$
\alpha[p] L^{\prime}\left(v_{l}+[v] H\right)+\alpha[v] H^{\prime}\left(p_{l}+[p] L\right)+\left(p_{l}+[p] L\right)[u] H^{\prime}
$$




$$
-\eta[p] L^{\prime \prime}\left(v_{l}+[v] H\right)-2 \eta[p][v] L^{\prime} H^{\prime}-\eta\left(p_{l}+[p] L\right)[v] H^{\prime \prime}-\beta[u]^{2}\left(H^{\prime}\right)^{2}=0 .
$$

To express equation (5.19) in terms of $H$, equation (5.12) implies:

$$
\begin{aligned}
& {[p] L \equiv \beta[u] H^{\prime}+[p] H,} \\
& {[p] L^{\prime} \equiv \beta[u] H^{\prime \prime}+[p] H^{\prime},} \\
& {[p] L^{\prime \prime} \equiv \beta[u] H^{\prime \prime \prime}+[p] H^{\prime \prime} .}
\end{aligned}
$$

Substituting equations (5.20) into (5.19) then yields:

$$
\begin{aligned}
\alpha\left(\beta[u] H^{\prime \prime}+[p] H^{\prime}\right)\left(v_{l}+[v] H\right)+\left(\alpha[v] H^{\prime}+[u] H^{\prime}\right)\left(p_{l}+\beta[u] H^{\prime}+[p] H\right) \\
-\eta\left(\beta[u] H^{\prime \prime \prime}+[p] H^{\prime \prime}\right)\left(v_{l}+[v] H\right)-2 \eta[v] H^{\prime}\left(\beta[u] H^{\prime \prime}+[p] H^{\prime}\right) \\
\quad-\eta\left(p_{l}+\beta[u] H^{\prime}+[p] H\right)[v] H^{\prime \prime}-\beta[u]^{2}\left(H^{\prime}\right)^{2}=0 .
\end{aligned}
$$

Combining like orders of derivatives in equation (5.21) produces:

$$
\begin{aligned}
& -\eta \beta[u] H^{\prime \prime \prime}\left(v_{l}+[v] H\right) \\
& +\left(\alpha \beta[u]\left(v_{l}+[v] H\right)-\eta[p]\left(v_{l}+[v] H\right)-2 \eta \beta[u][v] H^{\prime}-\eta\left(p_{l}+\beta[u] H^{\prime}+[p] H\right)[v]\right) H^{\prime \prime} \\
& +\alpha[p] H^{\prime}\left(v_{l}+[v] H\right)+(\alpha[v]+[u]) H^{\prime}\left(p_{l}+\beta[u] H^{\prime}+[p] H\right)-\left(2 \eta[p][v]+\beta[u]^{2}\right)\left(H^{\prime}\right)^{2}=0, \\
& -\eta \beta[u] H^{\prime \prime \prime}\left(v_{l}+[v] H\right) \\
& +\left(\alpha \beta[u] v_{l}-\eta[p] v_{l}-\eta[v] p_{l}\right) H^{\prime \prime}+(\alpha \beta[u][v]-2 \eta[p][v]) H H^{\prime \prime}-3 \eta \beta[v][u] H^{\prime} H^{\prime \prime} \\
& +\left(\alpha[p] v_{l}+\alpha[v] p_{l}+[u] p_{l}\right) H^{\prime}+(\alpha \beta[u][v]-2 \eta[p][v])\left(H^{\prime}\right)^{2} \\
& +\left(\alpha[p][v]+\frac{[u][p]}{2}\right)\left(H^{2}\right)^{\prime}=0,
\end{aligned}
$$

and collecting the terms for $\left(H H^{\prime}\right)^{\prime}$,

$$
\begin{aligned}
& -\eta \beta[u] H^{\prime \prime \prime}\left(v_{l}+[v] H\right) \\
& +\left(\alpha \beta[u] v_{l}-\eta[p] v_{l}-\eta[v] p_{l}\right) H^{\prime \prime}+(\alpha \beta[u][v]-2 \eta[p][v])\left(H H^{\prime}\right)^{\prime}-3 \eta \beta[v][u] H^{\prime} H^{\prime \prime} \\
& \quad+\left(\alpha[p] v_{l}+\alpha[v] p_{l}+[u] p_{l}\right) H^{\prime}+\left(\alpha[p][v]+\frac{[u][p]}{2}\right)\left(H^{2}\right)^{\prime}=0 .
\end{aligned}
$$

Now for the case of a non-heat conducting gas, set $k=0$ which implies $\eta=0$. Equation (5.22) then reduces to 


$$
\begin{aligned}
\alpha \beta[u] v_{l} H^{\prime \prime}+\alpha \beta[u][v]\left(H H^{\prime}\right)^{\prime}+ & \left(\alpha[p] v_{l}+\alpha[v] p_{l}+[u] p_{l}\right) H^{\prime} \\
& +\left(\alpha[p][v]+\frac{[u][p]}{2}\right)\left(H^{2}\right)^{\prime}=0 .
\end{aligned}
$$

Recalling equation (4.7) and

$$
\alpha=\frac{1}{\gamma-1} \cdot \frac{\tilde{u}_{l}}{v_{l}}
$$

imply that equation (5.23) may be written:

$$
\tilde{u}_{l} \beta[u] H^{\prime \prime}+\left(\tilde{u}_{l}[p]+\gamma p_{l}[u]\right) H^{\prime}+\beta[u]^{2}\left(H H^{\prime}\right)^{\prime}+\frac{\gamma+1}{2}[p][u]\left(H^{2}\right)^{\prime}=0 .
$$

Integrating the last expression yields:

$$
\tilde{u}_{l} \beta[u] H^{\prime}+\left(\tilde{u}_{l}[p]+\gamma p_{l}[u]\right) H+\beta[u]^{2} H H^{\prime}+\frac{\gamma+1}{2}[p][u] H^{2}=\kappa,
$$

where $\kappa$ is a constant of integration. The boundary conditions $H, H^{\prime}=0$ at $\xi=0$ then imply

$$
\kappa=0 \text {, }
$$

while the boundary conditions $H=1$ and $H^{\prime}=0$ at $\xi=\varepsilon$ give:

$$
\tilde{u}_{l}[p]+\gamma p_{l}[u]+\frac{\gamma+1}{2}[p][u]=0 \text {. }
$$

Example 28. Equation (5.27) may be shown to be true by rewriting this coefficient using equation (4.7) as:

$$
\frac{[p]}{p_{l}}+\gamma \frac{[v]}{v_{l}}+\frac{\gamma+1}{2} \frac{[p]}{p_{l}} \frac{[v]}{v_{l}}=0 .
$$

Combining equation (5.28) with the Rankine-Hugoniot equation (4.29) then yields

$$
1+\gamma\left(\frac{-2}{2 \gamma+(\gamma+1)[p] / p_{l}}\right)+\frac{\gamma+1}{2} \frac{[p]}{p_{l}}\left(\frac{-2}{2 \gamma+(\gamma+1)[p] / p_{l}}\right)=0,
$$

which implies

$$
2 \gamma+(\gamma+1)[p] / p_{l}-2 \gamma-(\gamma+1)[p] / p_{l}=0
$$

as claimed. 
Applying equation (5.26) to (5.25), the ordinary differential equation for the Heaviside function becomes:

$$
\left(\tilde{u}_{l} \beta[u]+\beta[u]^{2} H\right) H^{\prime}+\left(\tilde{u}_{l}[p]+\gamma p_{l}[u]+\frac{\gamma+1}{2}[p][u] H\right) H=0
$$

and

$$
\beta[u]\left(1+\frac{[v]}{v_{l}} H\right) H^{\prime}+p_{l}\left(\frac{[p]}{p_{l}}+\gamma \frac{[v]}{v_{l}}+\frac{\gamma+1}{2} \frac{[p]}{p_{l}} \frac{[v]}{v_{l}} H\right) H=0 .
$$

Finally, recasting equation (5.29) as an integral equation yields:

$$
\frac{[p]}{p_{l}} \int \frac{1+\frac{[v]}{v_{l}} H}{\left(\frac{[p]}{p_{l}}+\gamma \frac{[v]}{v_{l}}+\frac{\gamma+1}{2} \frac{[p]}{p_{l}} \frac{[v]}{v_{l}} H\right) H} d H=-\frac{[p]}{\beta[u]} \xi+\zeta,
$$

where $\zeta$ is a constant of integration.

To compute the Heaviside function for the specific volume, equation (5.30) was solved numerically. Since the left-hand-side of (5.30) is zero for $\xi=0$, the constant of integration becomes zero, $\zeta=0$. The coefficient $-\frac{[p]}{\beta[u]}$ appearing on the right-hand-side of equation (5.30) contains the viscosity of the gas and is a very large number which implies that the jump will occur over a very small spatial interval. Once equation (5.30) was solved for $H(\xi)$, equation (5.20) was applied to solve for $L(\xi)$.

Figures 5 and 6 compare the computed Heaviside functions for specific volume and pressure for the Mach numbers of 1.5 and 3.0. All calculations performed shown here assume $\gamma=\frac{7}{5}$. These figures show that the microstructure associated with the specific volume and the pressure is distinct and is a function of the Mach number of the flow field. A key result of the viscous analysis is that the equations of motion contain enough physical information to specify the jump functions $H, K$ and $L$. Figure 7 shows the specific volume jump function in terms of the Mach numbers 1.5, 2.0, 2.5 and 3.0; while, Figure 8 shows the pressure jump function for the same Mach numbers. 


\subsection{Jump Conditions for a Viscous Heat Conducting Gas}

The jump conditions for a viscous, heat conducting, perfect gas follow from the analysis of Section 5.1 with the minor modification that the coefficient of thermal conductivity $k$ is assumed to be a non-zero constant. Recalling that the functions $H(\xi), K(\xi)$, and, $L(\xi)$ are smooth nonstandard Heaviside functions with their jumps contained on the infinitesimal interval $(0, \varepsilon)$ for the specific volume, velocity and, pressure, respectively. The jump conditions for these field variables are governed by equations (5.5), (5.13), and (5.22):

$$
\begin{gathered}
H=K, \\
H(\xi)=\Phi \int_{0}^{\xi} \exp (\Phi\{\varsigma-\xi\}) L(\varsigma) d \varsigma,
\end{gathered}
$$

and

$$
\begin{aligned}
& -\eta \beta[u] H^{\prime \prime \prime}\left(v_{l}+[v] H\right) \\
& +\left(\alpha \beta[u] v_{l}-\eta[p] v_{l}-\eta[v] p_{l}\right) H^{\prime \prime}+(\alpha \beta[u][v]-2 \eta[p][v])\left(H H^{\prime}\right)^{\prime}-3 \eta \beta[v][u] H^{\prime} H^{\prime \prime} \\
& \quad+\left(\alpha[p] v_{l}+\alpha[v] p_{l}+[u] p_{l}\right) H^{\prime}+\left(\alpha[p][v]+\frac{[u][p]}{2}\right)\left(H^{2}\right)^{\prime}=0
\end{aligned}
$$

where

$$
\Phi \equiv \frac{[p]}{\beta[u]}, \beta \equiv \frac{4}{3} \mu, \eta=\frac{k}{R} \text {, and } \alpha=\frac{1}{\gamma-1} \cdot \frac{\tilde{u}_{l}}{v_{l}} .
$$

Equation (5.33) may be integrated once to yield a second order ODE for the specific volume jump function:

$$
\begin{aligned}
& -\eta \beta[u] H^{\prime \prime}\left(v_{l}+[v] H\right)-\eta \beta[u][v]\left(H^{\prime}\right)^{2} \\
& -\left(\eta[v] p_{l}-\alpha \beta[u] v_{l}+\eta[p] v_{l}-\alpha \beta[u][v] H+2 \eta[p][v] H\right) H^{\prime} \\
& \quad+\left([u] p_{l}+\alpha[v] p_{l}+\alpha[p] v_{l}\right) H+\frac{[p]}{2}([u]+2 \alpha[v]) H^{2}=\kappa .
\end{aligned}
$$

By applying the boundary conditions $H, H^{\prime}=0$ at $\xi=0$,

$$
\kappa=0 \text {, }
$$


while the boundary conditions $H=1$ and $H^{\prime}=0$ at $\xi=\varepsilon$ are satisfied since equation (5.34) reduces to equation (5.27). The boundary conditions for equation (5.34) then become:

$$
H(0)=0 \text { and } H(\varepsilon)=1 .
$$

Once equation (5.34) is integrated to determine the specific volume Heaviside function, $H$, the pressure Heaviside function is computed via equation (5.20):

$$
L \equiv \frac{\beta[u]}{[p]} H^{\prime}+H
$$

To compute $H$ using equation (5.34), the coefficients are grouped so that the physical parameters associated with the pressure and specific volume jumps across the shock wave may be expressed in terms of the Mach number by applying equations (4.32) and (4.33). The visocity and heat conduction parameters are introduced in the Prandlt number. The Prandlt number enters into equation (5.34) via the ratio of $\eta$ and $\beta$ :

$$
\delta \equiv \frac{\eta}{\beta}=\frac{k}{R} \cdot \frac{3}{4 \mu}=\frac{k}{R} \cdot \frac{3}{4 \mu} \cdot \frac{\gamma(\gamma-1)}{\gamma(\gamma-1)}=\frac{3}{4} \cdot \frac{\gamma}{(\gamma-1) \operatorname{Pr}} .
$$

Equation (5.30) for a viscous non-heat conducting gas, shows that the thickness of the shock depends on the parameter $\Phi \equiv \frac{[p]}{\beta[u]}$ containing the viscosity $\beta \equiv \frac{4}{3} \mu$. To remove this parameter from the governing equation, the following change of variables is introduced:

$$
\varsigma=-\Phi \xi
$$

so that

$$
\frac{d}{d \varsigma} \equiv()^{\prime}=-\frac{1}{\Phi} \frac{d}{d \xi}
$$

For derivation in this section the Prandlt number is fixed to be $\operatorname{Pr}=\frac{3}{4}$. So equation (5.36) implies:

$$
\delta=\frac{\gamma}{\gamma-1}
$$

Then applying equations (5.36), (5.37), and (5.38) to (5.34), the governing equation reduces to:

$$
\delta \frac{[p]}{p_{l}} H^{\prime \prime}+\delta \frac{[p]}{p_{l}} \frac{[v]}{v_{l}} H H^{\prime \prime}+\left(\left(\frac{1}{\gamma-1}+\delta\right) \frac{[p]}{p_{l}}+\delta \frac{[v]}{v_{l}}\right) H^{\prime}+\delta \frac{[p]}{p_{l}} \frac{[v]}{v_{l}}\left(H^{\prime}\right)^{2}
$$




$$
+\left(\frac{1}{2(\gamma-1)}+\delta\right) \frac{[p]}{p_{l}} \frac{[v]}{v_{l}} H H^{\prime}+\frac{1}{\gamma-1}\left(\frac{[p]}{p_{l}}+\gamma \frac{[v]}{v_{l}}\right) H-\left(\frac{1}{\gamma-1} \frac{\gamma+1}{2} \frac{[p]}{p_{l}} \frac{[v]}{v_{l}}\right)(H)^{2}=0 .
$$

To find the specific volume Heaviside function, $H(\xi)$, for a viscous, heat conducting gas, equation (5.39) must be solved numerically. To perform such calculations, the boundary conditions of (5.35) were replaced with initial conditions. The initial data may be specified from the physics of the flow field requiring that the jump function satisfy an isentropic compression as the flow enters the shock wave. In practice, this amounts to fixing the initial data as:

$$
H(0)=\vartheta \text { and } H^{\prime}(0)=\Im(M, \vartheta),
$$

where $\vartheta$ is a very small value. Numerically it was observed that as $M \rightarrow 1$ or if $M$ was fixed and $\vartheta \rightarrow 0$ the computed jump function $H(\xi)$ becomes symmetrical about an inflection point.

Figures 9, 10, 11 and 12 show Heaviside functions for specific volume, pressure, entropy and temperature, computed for a viscous, heat conducting gas as a function of the Mach numbers 1.5, 2.0, 2.5 and 3.0. As in the above section, all calculations shown assume $\gamma=\frac{7}{5}$. The temperature Heaviside function was computed from the specific volume and pressure jump functions using the equation of state (2.11). Figures 13 and 14 compare the microstructure of the field variables for the Mach numbers 1.5 and 3.0. The entropy for a viscous, heat conducting gas exhibits a peak as was predicted via the inviscid analysis. The microstructure peaks observed in the entropy and the temperature are the result of jump functions combined with nonlinear equations of motion.

\subsection{Comparison of Shock Wave Jump Conditions with Experimental Data}

To check the shock wave microstructure predicted by equation (5.39), the resulting specific volume jump function, $H(\xi)$ was compared with the density measured across a rarefied shock wave by Muntz and Harnett [39]. In the Muntz-Harnett experiment, a supersonic helium flow with a Mach number of 1.59 was used to generate a normal shock wave. The resulting normal shock wave microstructure was approximately $2.2 \mathrm{~cm}$ wide, a distance which represented 23-4 mean-free-paths for the HE molecules. The data produced in the experiment was also shown in reference [39] to be in good agreement with a computational solution of the Navier-Stokes equations. 
For the present comparison, the Prandlt number and ratio of specific heats were specified for helium as:

$$
\operatorname{Pr}=\frac{2}{3} \text { and } \gamma=\frac{5}{3} .
$$

Moreover, density was nondimensionalized using the following functional form:

$$
\rho^{*}=\frac{\rho-\rho_{l}}{\rho_{r}-\rho_{l}},
$$

with

$$
\rho_{l}=1.92 \times 10^{-8} \mathrm{~g} / \mathrm{cm}^{3} \text { and } \rho_{r}=3.51 \times 10^{-8} \mathrm{~g} / \mathrm{cm}^{3} .
$$

Substituting the computed specific volume Heaviside function into equation (5.41) then yielded:

$$
\rho^{*}=\frac{1 /\left(v_{l}+[v] H\right)-\rho_{l}}{\rho_{r}-\rho_{l}} .
$$

Figure 15 compares the measured shock wave microstructure with that predicted by equation (5.39). Overall the predicted density (specific volume) jump function is in good agreement with the measured density. The main variation observed occurred near $\rho^{*}=1$. The shape of the density jump function depends on the initial data, (5.40). As the initial data approached zero the predicted results produced were in good agreement with the experimental data. The predicted jump function matched the measured jump condition closely around $\rho^{*}=0$.

The microstructure computed for the other field variables for a normal helium shock wave is very similar to the jump functions shown in Figures 10, 11 and 12 of Section 5.3 for the $M=1.5$ case with $\operatorname{Pr}=\frac{3}{4}$ and $\gamma=\frac{7}{5}$ and, as such, is not shown. 


\subsection{SUMMARY AND CONCLUSIONS}

This report derived shock wave jump conditions for a one-dimensional compressible gas using nonstandard analysis. Differentiable internal Heaviside functions were introduced and used to model the microstructure of the flow field across a normal shock wave governed by the classical conservation equations. For flow variables with their jumps defined on the same infinitesimal interval, it was shown that the equations of motion written in nonconservative form produced unambiguous relations between the various nonstandard Heaviside functions. The analysis specified the microstructure across the shock wave for the cases in which the equations of motion contained a physical model of the shock region. For an inviscid shock wave, the analysis only produced the relations between the field variable Heaviside functions, because the equations of motion contained no physical information about the shock region. On the other hand for a viscous shock wave, the analysis predicted the microstructure across the shock wave. The entropy peak observed across a shock wave was predicted by both the inviscid and the viscous analyses and is the result of combining jump conditions with nonlinear field equations.

The present report was motivated by the authors' interest in understanding how to add physical information to specify products of generalized functions contained in differential equations modeling physical problems with well defined regions of solution discontinuity. The main conclusions of the report are as follows:

1. Nonstandard analysis may be used to identify differentiable internal Heaviside functions defined on infinitesimal intervals that reproduce the standard jump function results from distribution theory.

2. The derivative of a differentiable internal Heaviside function yields a predistribution of the Dirac delta measure.

3. Examples of differentiable internal Heaviside functions and pre-distributions of the delta measure were applied to compute nonstandard versions of products of generalized functions. The products of such generalized functions were shown to depend on the specific pre-distributions used to represent the

product. Higher order singular products such as $\delta^{2}$ in general do not yield standard generalized functions for functionals defined on spaces of standard 
test functions; although standard generalized function-like objects may result for functionals defined on spaces of nonstandard test functions.

4. Application of differentiable pre-distributions of the Heaviside function and the delta measure in products of generalized functions removes the algebraic over-specification and the associated mathematical ambiguities.

5. While mathematical ambiguities are removed by applying differentiable predistributions, additional physical information must be given to find problem specific pre-distributions for a given product of generalized functions. Products of generalized functions may be defined consistently via nonstandard analysis; however, physically meaningful products of generalized functions must be specified from the physics of the problem under investigation.

6. The relations between the internal Heaviside functions for a one-dimensional compressible inviscid gas were derived unambiguously from equations of motion in nonconservative from by using differentiable pre-distributions to represent the products of generalized functions.

7. The problem specific internal Heaviside functions for a one-dimensional compressible viscous gas were found (computed) unambiguously from equations of motion in nonconservative form by using differentiable predistributions to represent the products of generalized functions.

8. The shock wave jump conditions were computed directly by solving for the specific microstructure of the Heaviside functions of the flow parameters instead of the flow parameters themselves. The flow microstructure was defined on the characteristic lines as a traveling wave to transform the partial differential equations of motion to ordinary differential equations. The application of nonstandard analysis with the characteristic geometry of the flow produced a significant simplification of the problem, since the resulting ordinary differential equations are easier to solve than the equations of motion used in classical analyses. 


\subsection{REFERENCES}

1. Schwartz, L., "Sur l'impossibilite de la multiplication des distribution," Comptes Rendus de l'Academie des Sciences, Paris, 239, pp. 847-848, 1954.

2. Rosinger, E. E., Distributions and Nonlinear Partial Differential Equations, Lecture Notes in Mathematics, 684, Springer-Verlag, 1978.

3. Rosinger, E. E., Nonlinear Partial Differential Equations: Weak and Sequential Solutions, North-Holland Mathematics Studies, 44, Elsevier Science Publishers, 1980.

4. Rosinger, E. E., Generalized Solutions of Nonlinear Partial Differential Equations, NorthHolland Mathematics Studies, 146, Elsevier Science Publishers, 1987.

5. Rosinger, E. E., Non-Linear Partial Differential Equations: An Algebraic View of Generalized Solutions, North-Holland Mathematics Studies, 164, Elsevier Science Publishers, 1990.

6. Colombeau, J. F., New Generalized Functions and Multiplication of Distributions, NorthHolland Mathematics Studies, 84, Elsevier Science Publishers, 1984.

7. Colombeau, J. F., Elementary Introduction to New Generalized Functions, North-Holland Mathematics Studies, 113, Elsevier Science Publishers, 1985.

8. Rosinger, E. E., "Characterization for the Solvability of Nonlinear Partial Differential Equations," Transactions of the American Mathematical Society, 330, No. 1, pp. 203-225, 1992.

9. Colombeau, J. F., "The Elastoplastic Shock Problem as an Example of the Resolution of Ambiguities in the Multiplication of Distributions," Journal of Mathematical Physics, 30, No. 10, pp. 2273-2279, 1989.

10. Colombeau, J. F., and Le Roux, A. Y., "Multiplication of Distributions in Elasticity and Hydrodynamics," Journal of Mathematical Physics, 29, No. 2, pp. 315-319, 1988.

11. Colombeau, J. F., Le Roux, A. Y., Noussair, A., Perrot, B., "Microscopic Profiles of Shock Waves and Ambiguities in Multiplication of Distributions," SIAM Journal of Numerical Analysis, 26, No. 4, pp. 871-883, 1989.

12. Todorov, T. D., "Colombeau's Generalized Functions and Nonstandard Analysis," in Generalized Functions, Convergence Structures, and Their Applications, Edited by B. Stankovic, et al., Plenum Press, pp. 327-339, 1988. 
13. Oberguggenberger, M., "Products of Distributions: Nonstandard Methods," Zeitschrift fur Analysis und ihre Anwendunge, 7, pp. 347-365, 1988.

14. Hoskins, R. F. and Sousa Pinto, J., "Nonstandard Treatment of New Generalized Functions," in Generalized Functions and Their Application, Edited by Pathak, R. S., Plenum Press, pp. 95-103, 1993.

15. Robinson, A. Nonstandard Analysis, North-Holland, 1966.

16. Colombeau, J. F, Multiplication of Distributions, Lecture Notes in Mathematics, 1532, Springer-Verlag, 1992.

17. Morduchow, M. and Libby, P. A., "On the Distribution of Entropy Through a Shock Wave," Journal de Mecanique, 4, No. 2, pp. 191-213, 1965.

18. Salas, M. D. and Iollo, A., "Entropy Jump Across an Inviscid Shock Wave," Theoretical and Computational Fluid Dynamics, 8, pp. 365-375, 1996.

19. Baty, R. S., Vaughn, M. R., and Farassat, F., "A Nonstandard Analysis of a Simple Discontinuous Force Equation Modelling Continuous Motion," Journal of Sound and Vibration, 202, No. 2, pp. 288-297, 1997.

20. Keisler, H. J., Elementary Calculus: An Infinitesimal Approach, Prindle, Weber and Schmidt, $2^{\text {nd }}$ Edition, 1986.

21. Laugwitz, D., The Theory of Infinitesimals, An Introduction to Nonstandard Analysis, Accademia Nazionale Dei Lincei, 1980.

22. Currie, I. G., Fundamental Mechanics of Fluids, 3rd Edition, Marcel Dekker, Inc., 2003.

23. Thompson, P. A., Compressible-Fluid Dynamics, McGraw-Hill, 1972.

24. Hurd, A. E., Loeb, P. A., An Introduction to Nonstandard Real Analysis, Academic Press, 1985.

25. Henle, J. M. and Kleinberg, E., Infinitesimal Calculus, MIT Press, 1980.

26. Keisler, H. J., Foundations of Infinitesimal Calculus, Prindle, Weber and Schmidt, 1976.

27. Hoskins, R. F., Standard and Nonstandard Analysis, Ellis Horwood, 1990.

28. Lindstrom, T., "An Invitation to Nonstandard Analysis," Non-Standard Analysis and its Applications, London Mathematical Society Student Texts, 10, Edited by Cutland, N., Cambridge University Press, pp. 1-105, 1988.

29. Stroyan, K. D. and Luxemburg, W. A. J., Introduction to the Theory of Infinitesimals, Academic Press, 1976. 
30. Davis, M., Applied Nonstandard Analysis, John Wiley \& Sons, 1977.

31. Arkeryd, L. O., Cutland, N. J., and Henson, C. W., Nonstandard Analysis Theory and Applications, NATO ASI Series, Series C: Mathematical and Physical Sciences, 493, Kluwer Academic Publishers, 1997.

32. Hungerford, T. W., Algebra, Springer-Verlag, 1974.

33. Goldblatt, R, Lectures on the Hyperreals: An Introduction to Nonstandard Analysis, Springer-Verlag, 1998.

34. Keleman, P. J., and Robinson, A., "The Nonstandard $\lambda: \phi_{2}^{4}(x)$ : Model. I. The Technique of Nonstandard Analysis in Theoretical Physics," Journal of Mathematical Physics, 13, No. 12, pp. 1870-1874, December 1972.

35. Laugwitz, D., "Infinitesimals in Physics (An Introduction to the Application of Nonstandard Methods)," Mathematical Structures -- Computational Mathematics -- Mathematical Modelling, 2, Papers dedicated to Professor L. Iliev's 70th Anniversary, Sofia, pp. 233-243, 1984.

36. Richter, M. M., Ideale Punkte, Monaden und Nichstandard-Methoden, Friedr. Vieweg \& Sohn, 1982.

37. Palmgren, E., "Constructive Nonstandard Representations of Generalized Functions," Indagationes Mathematicae New Series, 11, No. 1, pp. 129-138, 2000.

38. Baty, R. S., Nonlinear Generalized Functions and Nonconservative Shock Simulations, SAND94-2258, Sandia National Laboratories, 1994.

39. Muntz, E. P., and Harnett, L. N., "Molecular Velocity Distribution Function Measurements in a Normal Shock Wave,” The Physics of Fluids, 12, No. 10, pp. 2027-2035, 1969. 


\subsection{FIGURES}

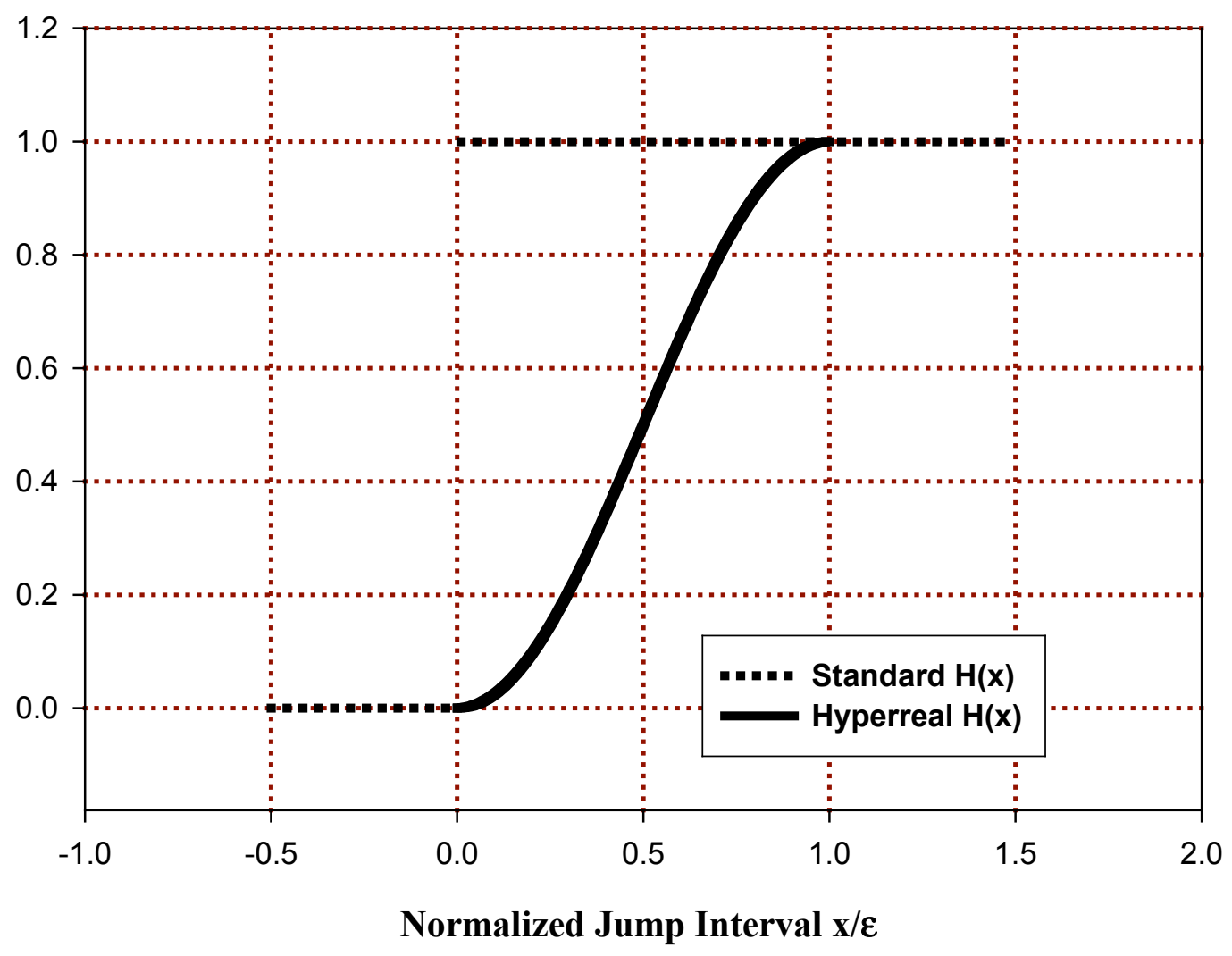

Figure 1: Comparison of standard and nonstandard Heaviside functions. The normalized jump interval is the unit interval $[0,1]$. 


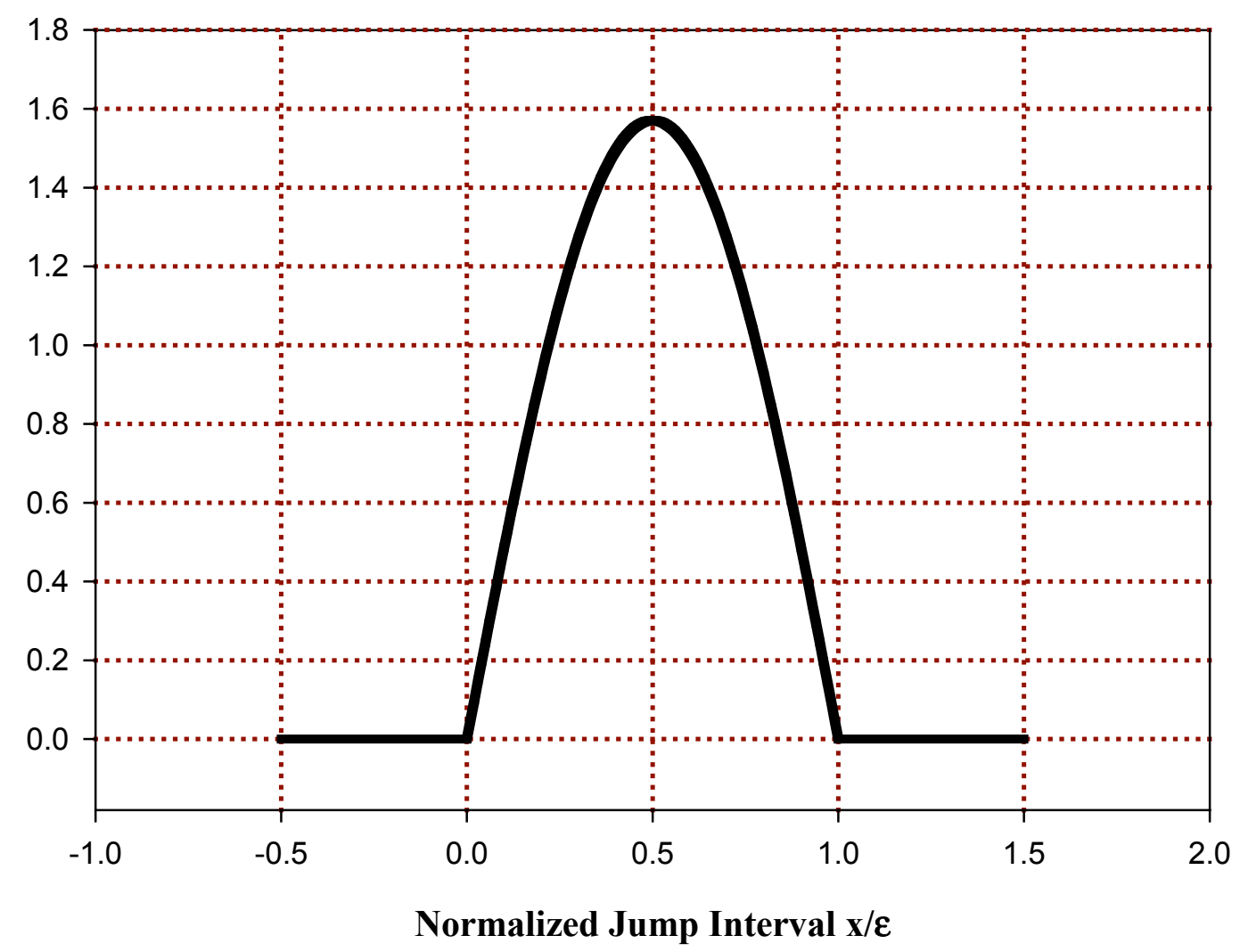

Figure 2: The derivative of the nonstandard Heaviside function shown in Figure 1 plotted on the normalized jump interval. 


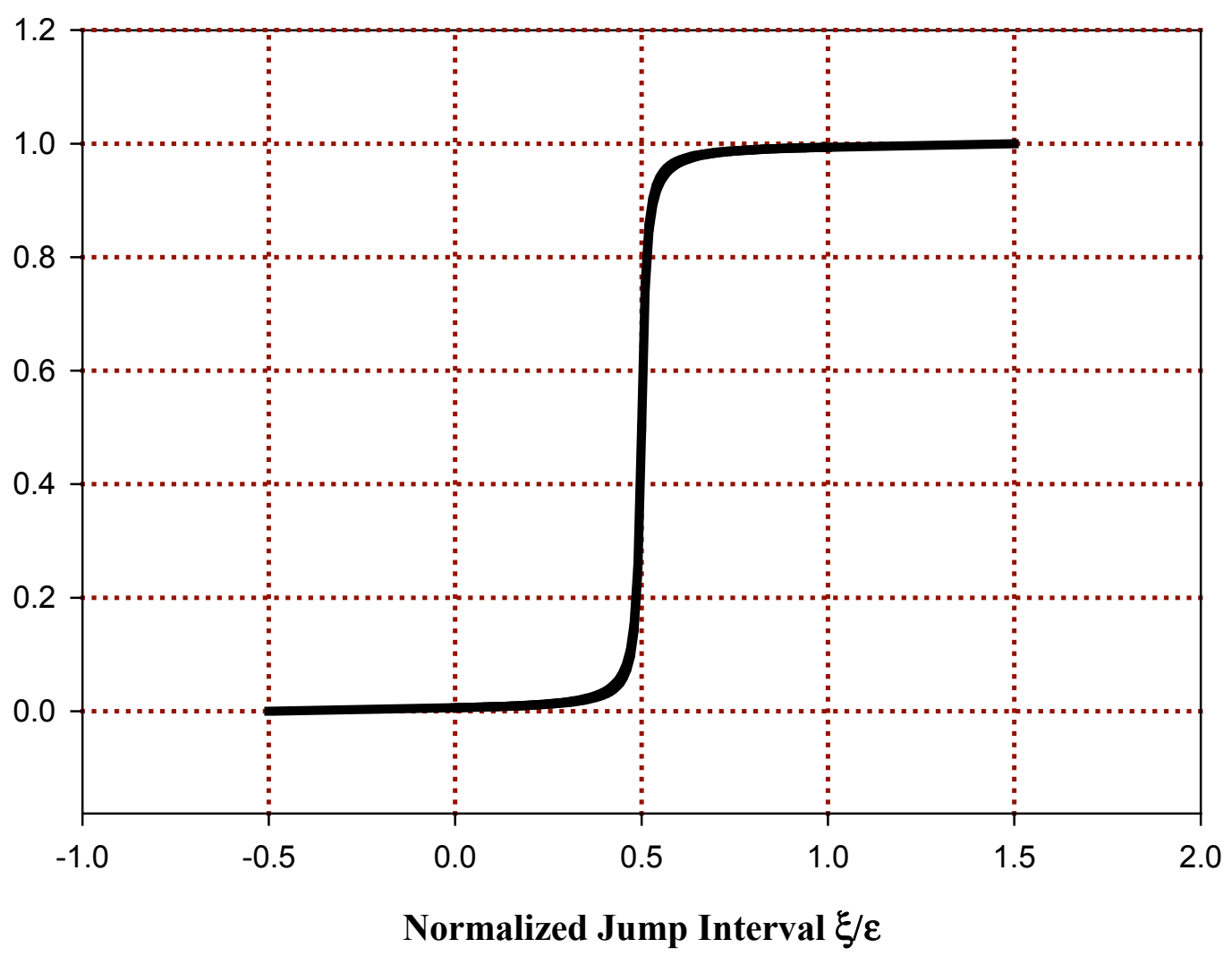

Figure 3: A smooth Heaviside function based on the inverse tan function plotted on the normalized jump interval with $\Omega=100$. 


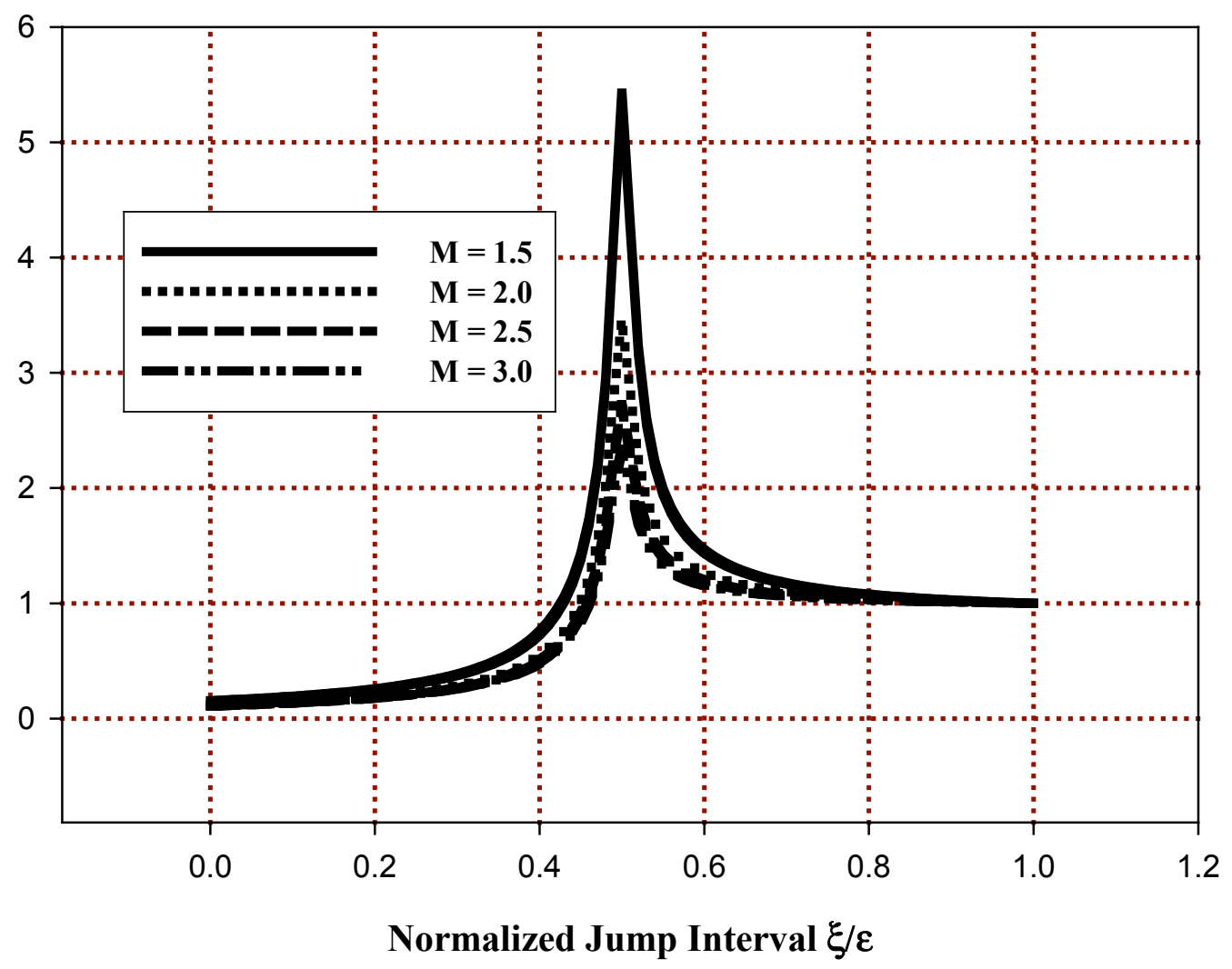

Figure 4: The inviscid entropy Heaviside function as a function of the Mach number plotted on the normalized jump interval. 


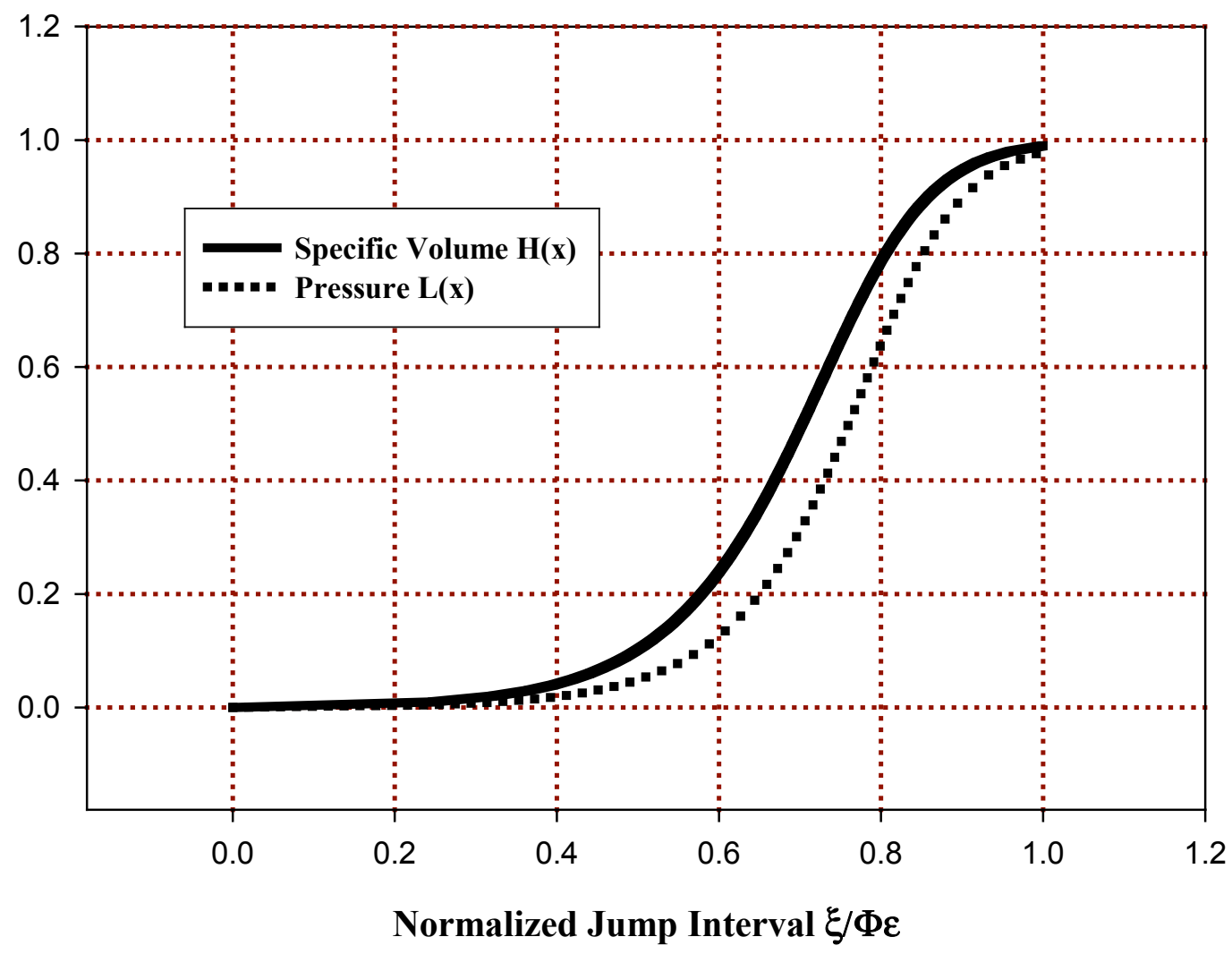

Figure 5: The viscous specific volume and pressure Heaviside functions for the Mach number 1.5 , plotted on the normalized jump interval. 


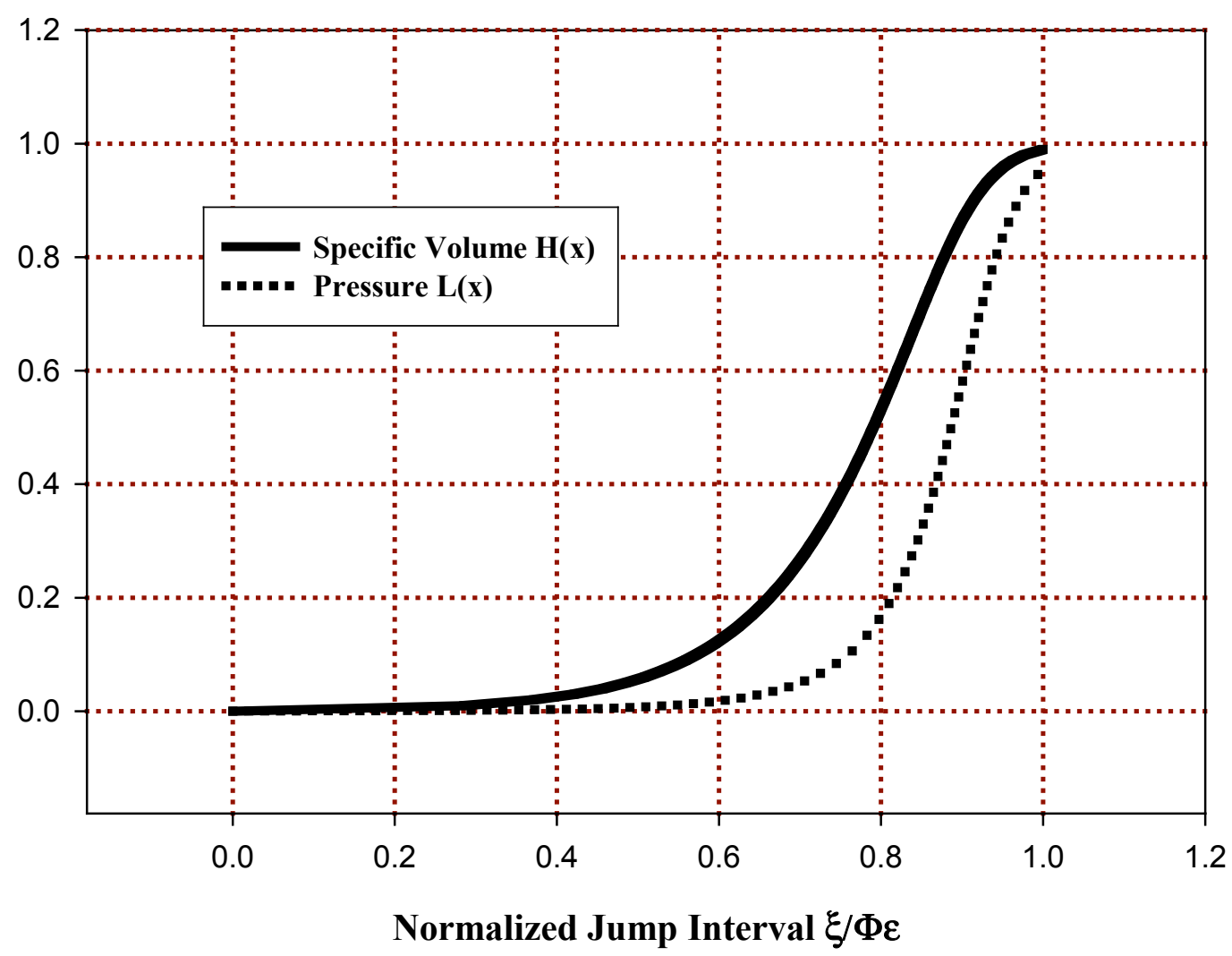

Figure 6: The viscous specific volume and pressure Heaviside functions for the Mach number 3.0, plotted on the normalized jump interval. 


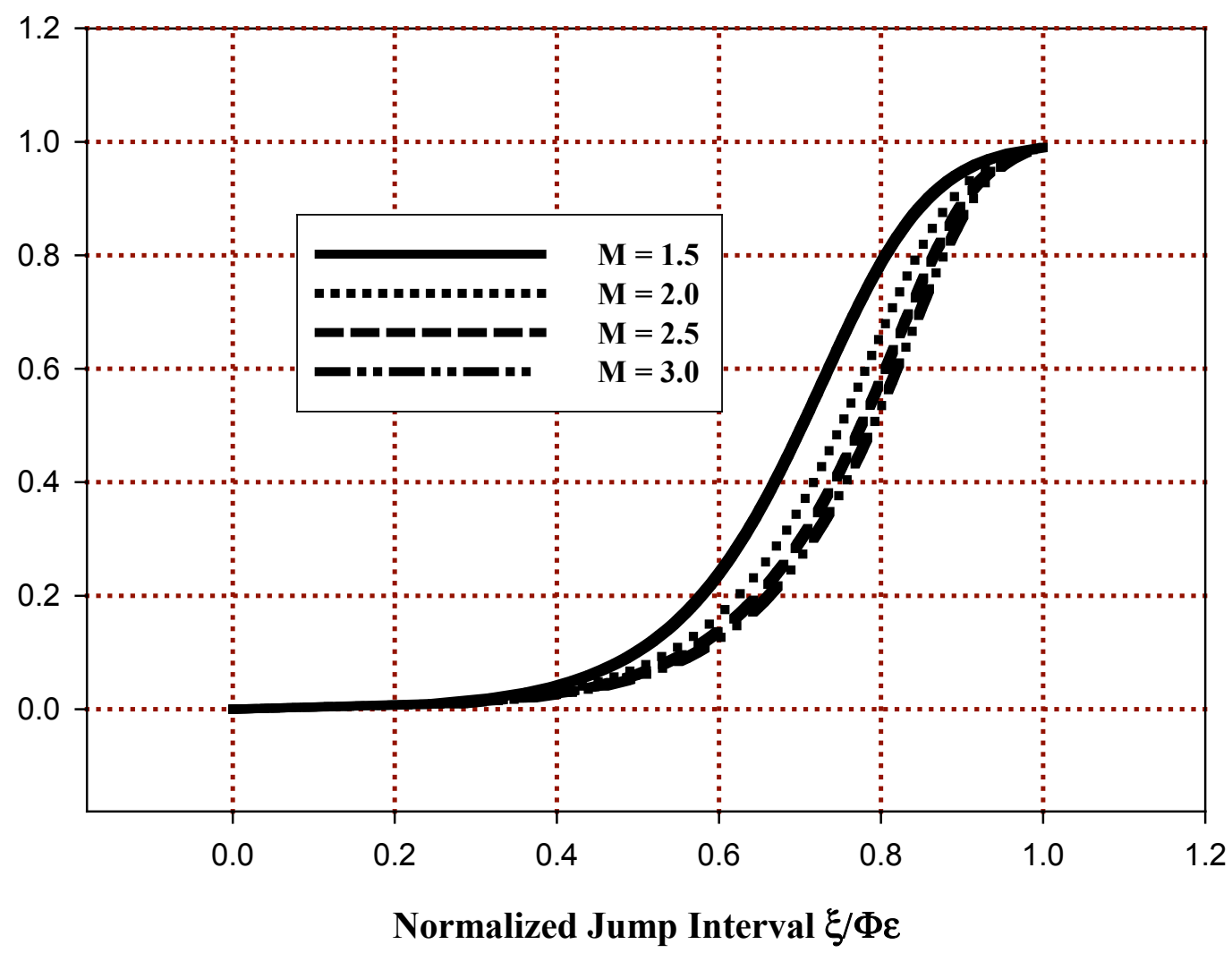

Figure 7: The viscous specific volume Heaviside function as a function of Mach number plotted on the normalized jump interval. 


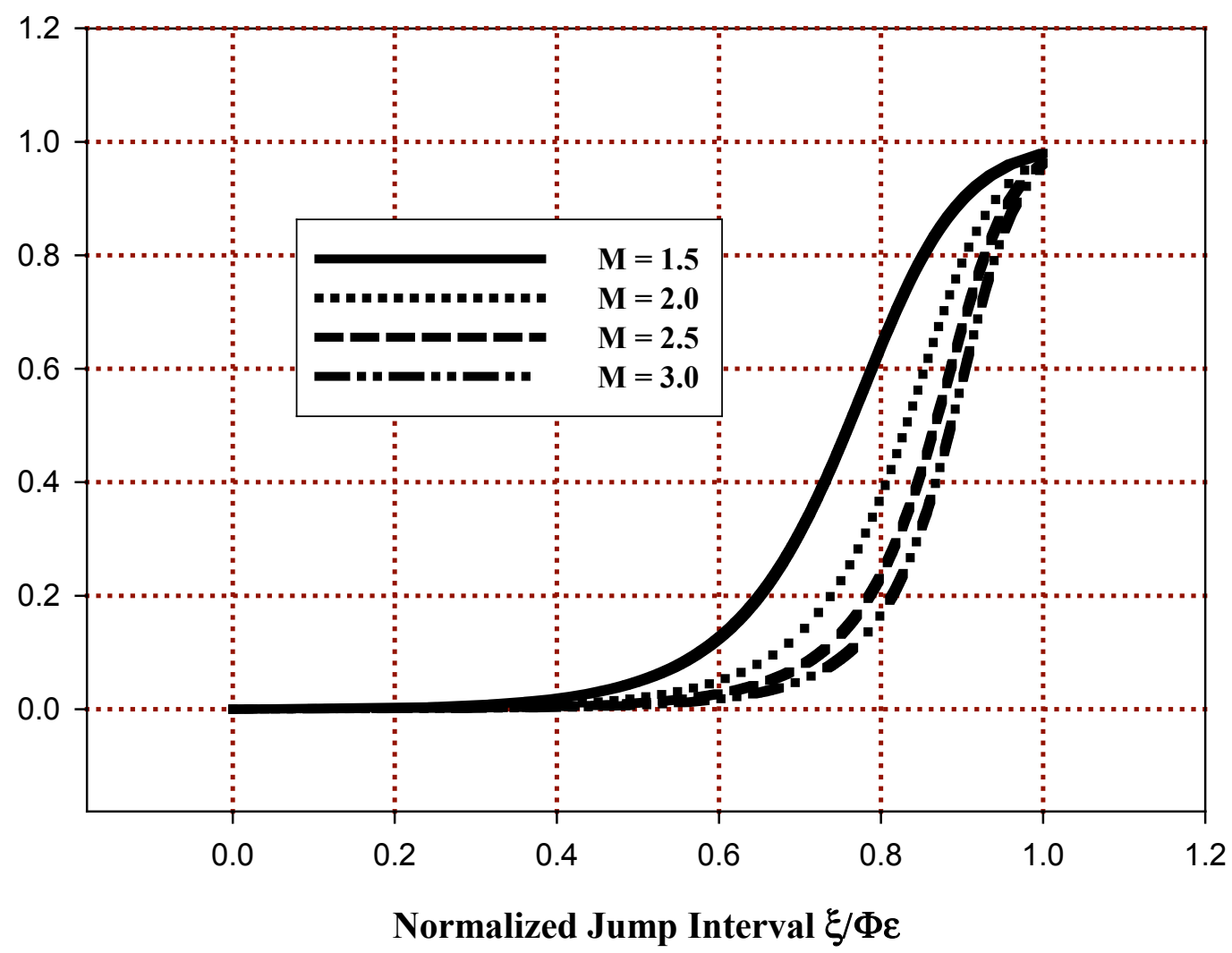

Figure 8: The viscous pressure Heaviside function as a function Mach number plotted on the normalized jump interval. 


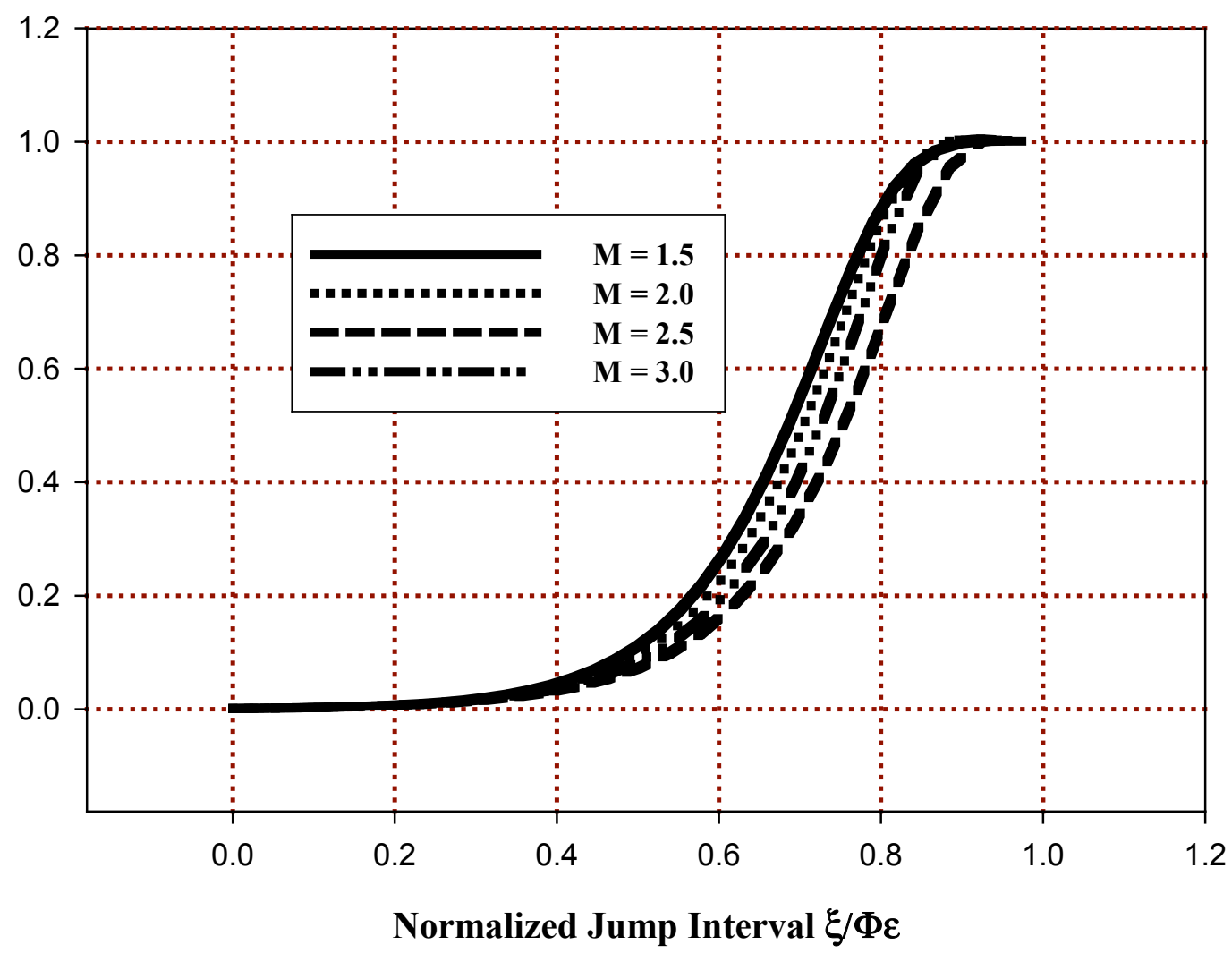

Figure 9: The viscous, heat conducting, specific volume Heaviside function as a function of Mach number plotted on the normalized jump interval. 


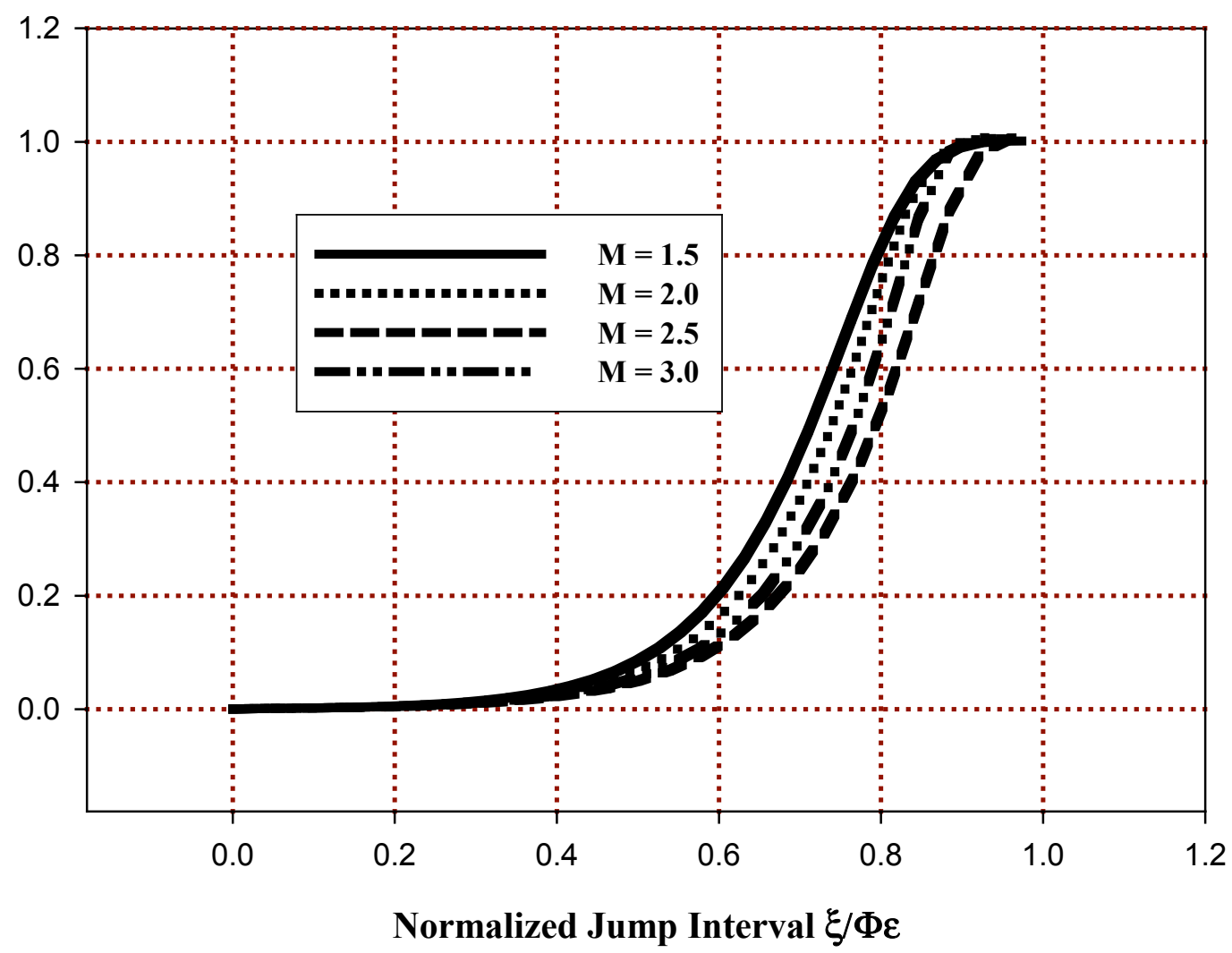

Figure 10: The viscous, heat conducting, pressure Heaviside function as a function of Mach number plotted on the normalized jump interval. 


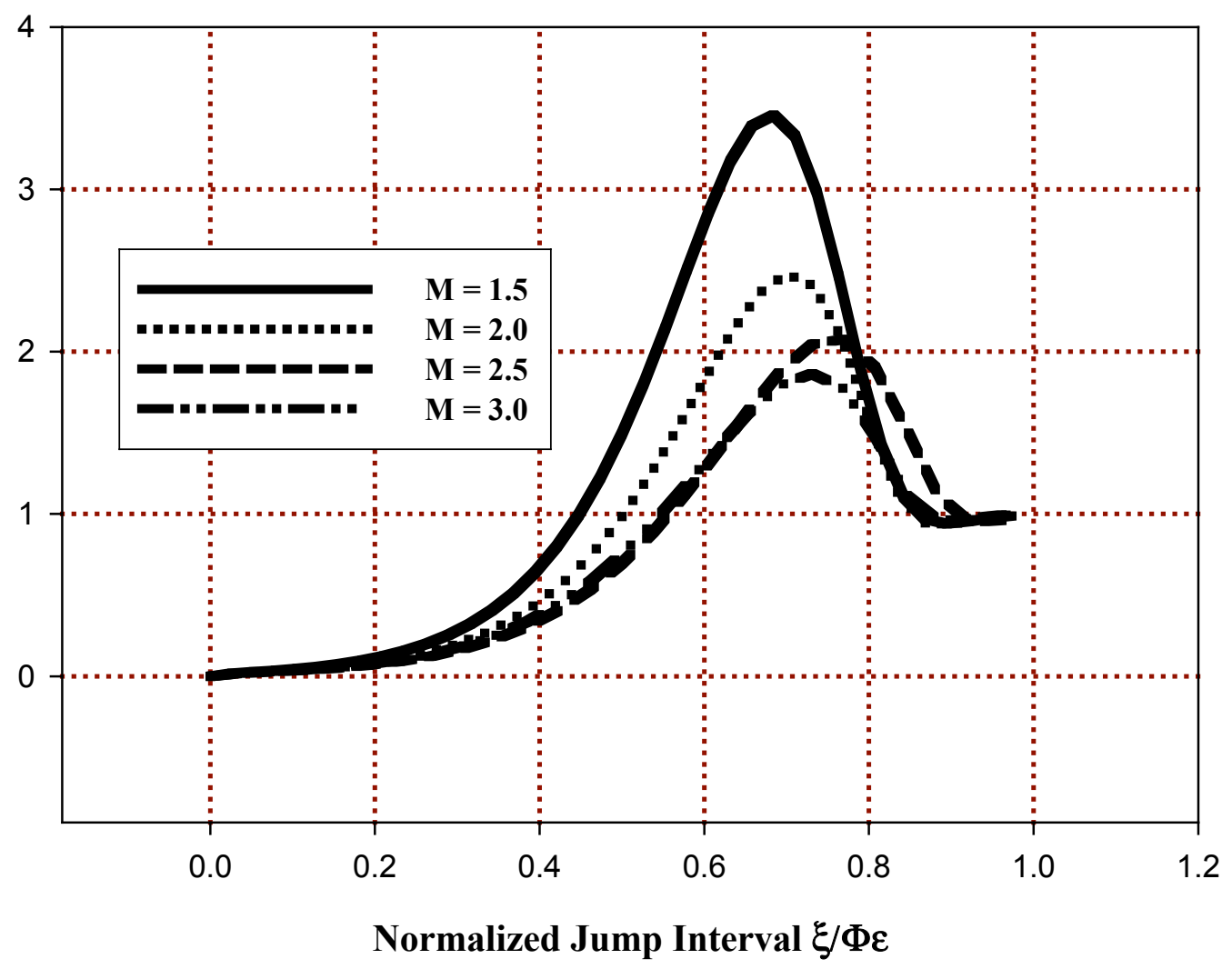

Figure 11: The viscous, heat conducting, entropy Heaviside function as a function of Mach number plotted on the normalized jump interval. 


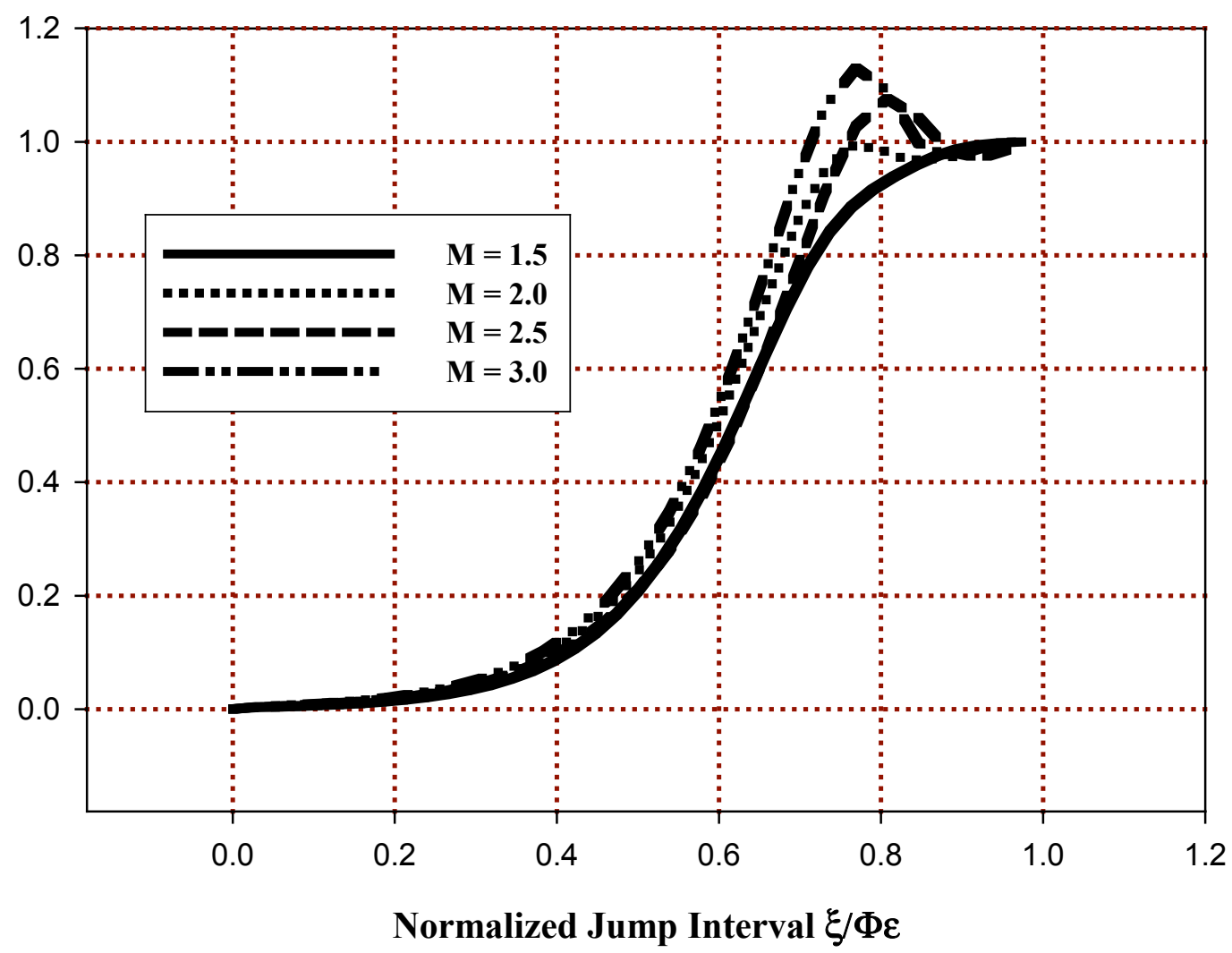

Figure 12: The viscous, heat conducting, temperature Heaviside function as a function of Mach number plotted on the normalized jump interval. 


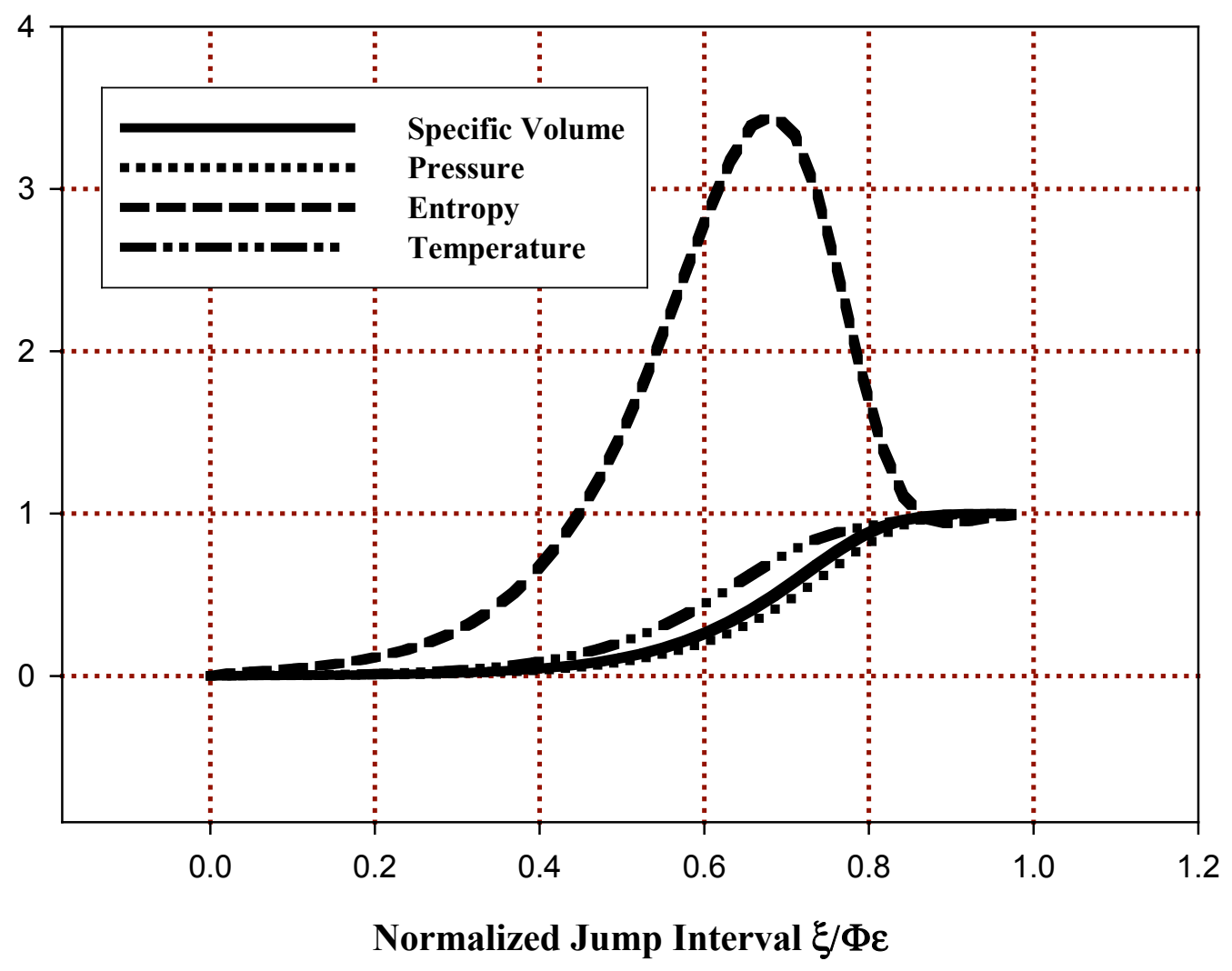

Figure 13: The viscous, heat conducting, field variable Heaviside functions for the Mach number 1.5 , plotted on the normalized jump interval. 


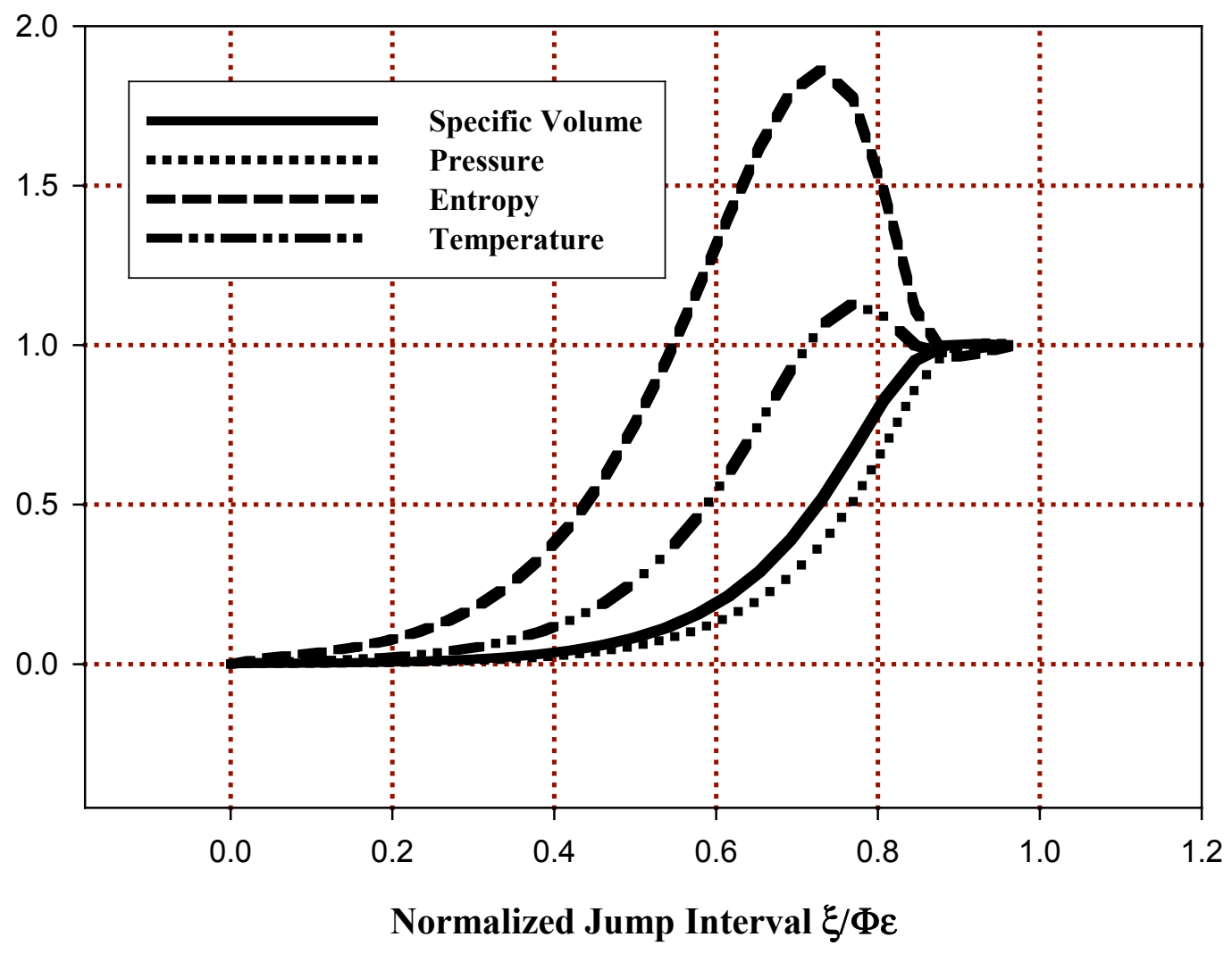

Figure 14: The viscous, heat conducting, field variable Heaviside functions for the Mach number 3.0, plotted on the normalized jump interval. 


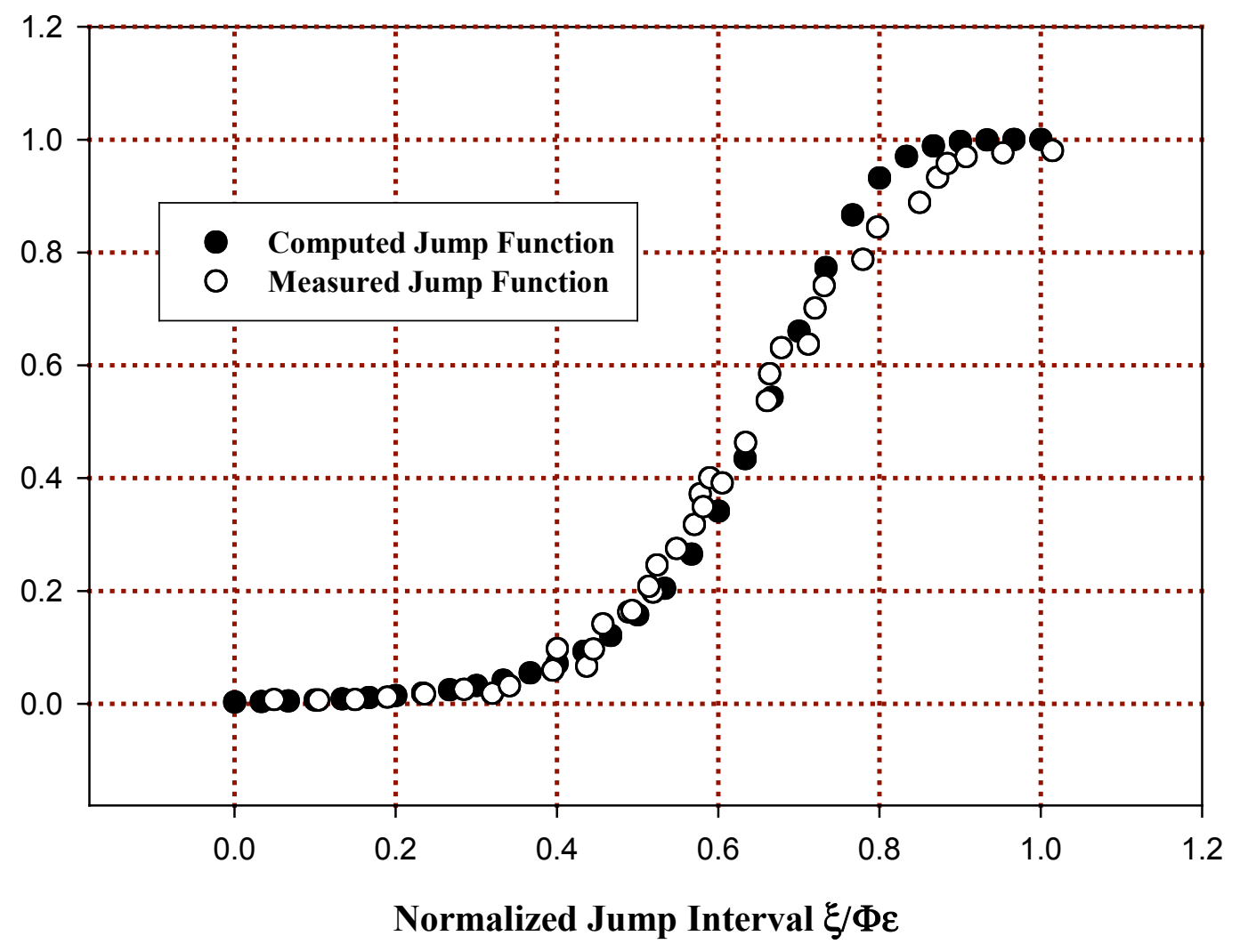

Figure 15: The comparison of the density microstructure computed with Equation (5.39) with the density microstructure measured for a Mach number 1.59, normal, rarefied helium shock wave. 
This report has been reproduced directly from the best available copy. It is available electronically on the Web (http://www.doe.gov/bridge).

Copies are available for sale to U.S. Department of Energy employees and contractors from:

Office of Scientific and Technical Information

P.O. Box 62

Oak Ridge, TN 37831

(865) 576-8401

Copies are available for sale to the public from: National Technical Information Service

U.S. Department of Commerce

5285 Port Royal Road

Springfield, VA 22161

(800) 553-6847 
- Los Alamos NATIONAL LABORATORY

EST.1943 